\title{
Twelve-year survival and immune correlates in dendritic cell-vaccinated melanoma patients
}

Stefanie Gross, ${ }^{1}$ Michael Erdmann, ${ }^{1}$ Ina Haendle, ${ }^{1}$ Steve Voland, ${ }^{1}$ Thomas Berger, ${ }^{1}$ Erwin Schultz, ${ }^{1}$ Erwin Strasser, ${ }^{2}$ Peter Dankerl, ${ }^{3}$ Rolf Janka, ${ }^{3}$ Stefan Schliep, ${ }^{1}$ Lucie Heinzerling, ${ }^{1}$ Karl Sotlar, ${ }^{4}$ Pierre Coulie, ${ }^{5}$ Gerold Schuler, ${ }^{1}$ and Beatrice Schuler-Thurner ${ }^{1}$

'Department of Dermatology, ${ }^{2}$ Department of Transfusion Medicine and Hemostaseology, ${ }^{3}$ Department of Radiology, University Hospital Erlangen, Friedrich-Alexander-University Erlangen-Nuremberg (FAU), Erlangen, Germany. ${ }^{4}$ Institute of Pathology, Ludwig-Maximilians-University, Munich, Germany. ${ }^{5}$ de Duve Institute, Université catholique de Louvain, Brussels, Belgium.

BACKGROUND. Reports on long-term ( $\geq 10$ years) effects of cancer vaccines are missing. Therefore, in 2002, we initiated a phase $\mathrm{I} / \mathrm{II}$ trial in cutaneous melanoma patients to further explore the immunogenicity of our DC vaccine and to establish its long-term toxicity and clinical benefit after a planned 10 -year followup.

METHODS. Monocyte-derived DCs matured by TNF $\alpha$, IL-1及, IL-6, and PCE ${ }_{2}$ and then loaded with 4 HLA class I and 6 class II-restricted tumor peptides were injected intradermally in high doses over 2 years. We performed serial immunomonitoring in all 53 evaluable patients.

RESULTS. Vaccine-specific immune responses including high-affinity, IFN $\gamma$-producing $\mathrm{CD4}^{+}$and lytic polyfunctional CD8 ${ }^{+} \mathrm{T}$ cells were de novo induced or boosted in most patients. Exposure of mature DCs to trimeric soluble CD40 ligand, unexpectedly, did not further enhance such immune responses, while keyhole limpet hemocyanin (KLH) pulsing to provide unspecific CD4+ help promoted CD8 ${ }^{+} \mathrm{T}$ cell responses - notably, their longevity. An unexpected $19 \%$ of nonresectable metastatic melanoma patients are still alive after 11 years, a survival rate similar to that observed in ipilimumab-treated patients and achieved without any major (>grade 2) toxicity. Survival correlated significantly with the development of intense vaccine injection site reactions, and with blood eosinophilia after the first series of vaccinations, suggesting that prolonged survival was a consequence of $\mathrm{DC}$ vaccination.

CONCLUSIONS. Long-term survival in advanced melanoma patients undergoing DC vaccination is similar to ipilimumab-treated patients and occurs upon induction of tumor-specific $\mathrm{T}$ cells, blood eosinophilia, and strong vaccine injection site reactions occurring after the initial vaccinations.

TRIAL REGISTRATION. ClinicalTrials.gov NCT00053391.

FUNDING. European Community, Sixth Framework Programme (Cancerimmunotherapy LSHCCT-2006-518234; DC-THERA LSHB-CT-2004-512074), and German Research Foundation (CRC 643, (1, Z2).

Role of funding source:

Authorship note: SG and ME contributed equally to this work.

Conflict of interest: The authors have declared that no conflict of interest exists.

Submitted: October 27, 2016

Accepted: March 2, 2017

Published: April 20, 2017

Reference information:

JCI Insight. 2017;2(8):e91438. https:// doi.org/10.1172/jii.insight.91438.

\section{Introduction}

The remarkable clinical success of checkpoint blockade antibodies (with cutaneous malignant melanoma as a paradigm) and the overall limited clinical impact of cancer vaccines have dampened interest in vaccination. However, as resistance to checkpoint blockade has become clinical reality and combination strategies are increasingly considered, there is a renewed interest in cancer vaccination $(1,2)$. Cancer vaccines aim at inducing or restimulating tumor-specific $\mathrm{T}$ cell responses. Based on preclinical (1-9) and initial clinical reports (10-12), they could be instrumental in breaking primary resistance to checkpoint blockade of tumors with low mutational load (13-15) and in increasing response rates of highly mutated tumors, such as melanoma (11). 
To induce or expand tumor-specific T cells, cancer vaccines must ensure that tumor antigens are presented by mature immunogenic DCs (16). This can be achieved, in principle, using three approaches (17), namely by (i) injecting antigen plus adjuvant in order to load and mature DCs in vivo, by (ii) targeted delivery of antigens (and ideally also adjuvants) to subsets of DCs in vivo, and (iii) by adoptive transfer of DCs that have been matured/activated and loaded with antigen ex vivo before injection into the patient. So far, only the third strategy (i.e., DC vaccination) has been successful in significantly improving overall survival (OS) without major side effects (despite the lack of an effect on progression-free survival) in 2 consecutive phase III trials (18-20). The respective cellular product (Sipuleucel-T) was approved by the FDA in 2010 for the treatment of asymptomatic or minimally symptomatic metastatic castration-resistant prostate cancer but has failed commercially. Failure was likely due to the logistical drawback that one apheresis was required for the production of each dose of this cellular vaccine. Rather than relying on rare preexisting DCs isolated from blood, we have been pursuing monocyte-derived DCs as a vaccine product, since mature monocyte-derived DCs can be reliably produced in large numbers from a single apheresis $(21,22)$ and have already proven immunogenic upon their initial use (23-26).

Here, we report on a monocentric clinical phase I/II trial in patients suffering from metastatic cutaneous melanoma. Besides evaluation of safety, tolerability, and clinical impact of the vaccination with a planned 10-year followup, the trial was designed with 2 cohorts, to compare the impact of 2 different DC activation strategies on the immunogenicity of the vaccine. In the first cohort, the matured monocytederived DCs were exposed to soluble bioactive trimeric CD40 ligand (CD40L, also known as CD154) (27, 28), to mimic T cell help and allow DC licensing for optimized induction of CD8 ${ }^{+}$cytotoxic T cells, even in the absence of cognate T cell help $(29,30)$. In the second cohort, all DCs that were pulsed with HLA class I-restricted tumor peptides (see Supplemental Table 3) were additionally loaded with keyhole limpet hemocyanin $(\mathrm{KLH})$ to provide unspecific $\mathrm{CD}^{+} \mathrm{T}$ cell help.

\section{Results}

Patients. A total of 62 eligible HLA-A1- and/or HLA-A2.1-positive advanced melanoma patients were included between 2002 and 2005 at the Department of Dermatology, FAU. Nine patients dropped out (either apheresis was not possible or patients progressed rapidly and did not reach the second apheresis to become evaluable). All remaining 53 patients received vaccinations \#1 to \#4 (Figure 1) and were thus fully evaluable for immunological response and toxicity as defined in the trial protocol. All 53 patients also proceeded to the second, maintenance part of the trial, in which - as per protocol - a total number of 6 vaccinations at increasing intervals for up to 2 years was delivered (Figure 1). Supplemental Table 1 (supplemental material available online with this article; https://doi.org/10.1172/jci.insight.91438DS1) depicts the total number of vaccinations administered to each patient, as well as information on patient characteristics.

Immunogenicity and characterization of vaccine-induced immune responses. To detect relevant tumor antigenspecific T cells with high sensitivity and to understand their kinetics, we performed serial in vitro-stimulated IFN $\gamma$ ELIspots in each patient at many time points. Figure 2 presents coherent images of these vitro-stimulated ELIspot (ivsELIspot) responses over time (prevaccination, induction, and maintenance phase of the trial) of all evaluable patients for each tumor peptide (Figure 2A). It also presents responses of cohort 1 (i.e., \pm CD40L pulsing of class I or II peptide-loaded DCs) or cohort 2 (i.e., \pm KLH loading of class I peptideloaded DCs) (Figure 2B). Data (Figure 2A) clearly reveal that preexisting tumor-specific responses are low or absent at the beginning, except those for the HLA-A2/Melan-A epitope, for which precursors are known to be frequent even in healthy individuals $(31,32)$. Upon vaccination, responses substantially increase but tend to be slightly lower in the maintenance phase of the trial, likely due to the increasing vaccine intervals (Figure 1). Indeed, depicting ELIspot data at each time point in individual patients illustrates not only that immunity is enhanced rapidly, as already observed in our previous trials $(24,26,33)$, but also that responses measured on the day of subsequent vaccinations decrease as soon as the interval to the preceding vaccination exceeds 6 weeks but do not completely vanish, indicating the formation of a pool of memory $\mathrm{T}$ cells (Supplemental Figure 1 and Supplemental Figure 7, A and B). Also of note was that, compared with HLAA2 peptides, HLA-A1-restricted peptides appeared much less immunogenic in the study population (Figure 2A), although, in individual patients, significant responses occurred (Supplemental Figure 1). It was also obvious (Figure 2B) that presenting class I- or class II-restricted peptides on CD40L-pulsed mature DCs in cohort 1 (patient \#01-30 and \#32) did not enhance immune responses over those achieved by non-CD40Lexposed cocktail-matured DCs, which was unexpected, given the known DC-activating effects of CD40 trig- 
A Induction phase Maintenance phase

(n)

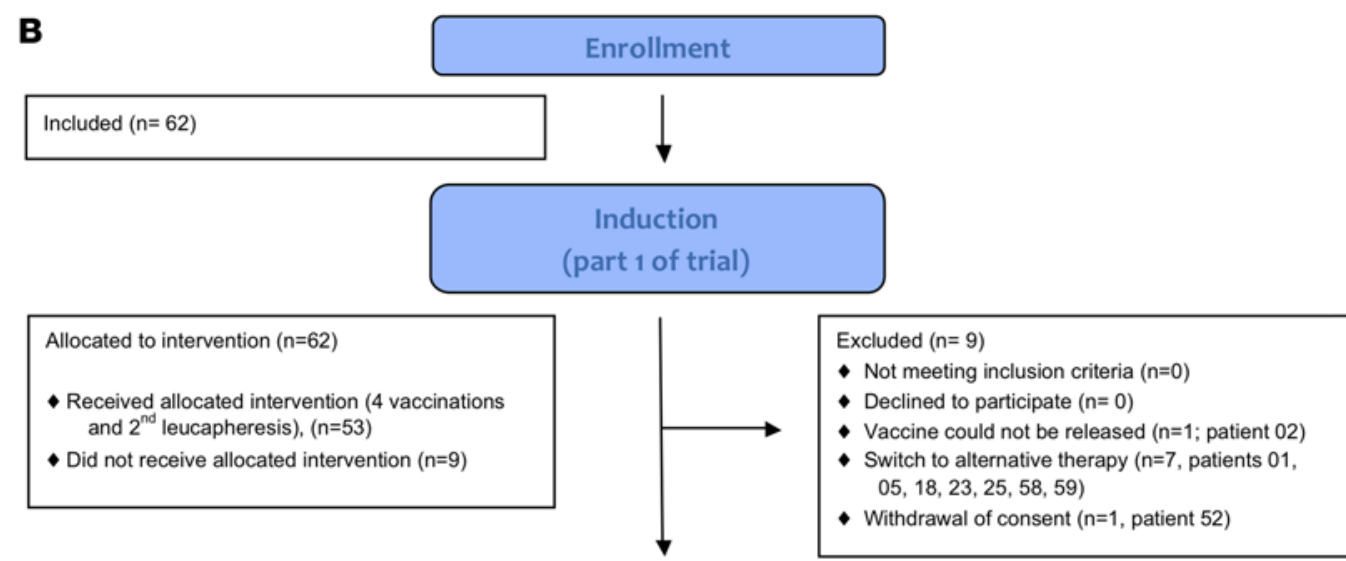

Maintenance

(part 2 of trial)

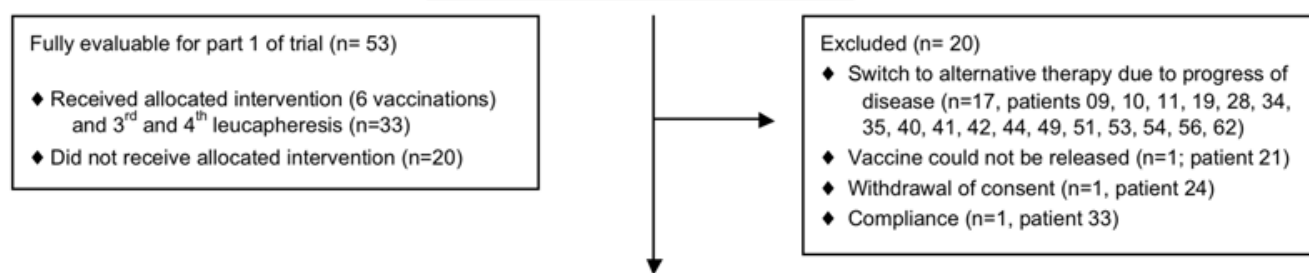

Trial completion

Fully evaluable for part 2 of trial $(n=33)$

Figure 1. Study scheme. (A) Leukaphereses were performed before trial start and then repeated after 3 months, 1 year, and 2 years. Vaccinations were given in increasing intervals of 2 weeks to 6 months. Clinical evaluations were performed every 3 months. (B) CONSORT 2010 flow chart describing patient numbers for enrollment, induction, and maintenance phase of the trial, as well as trial completion.

gering (27-30, 34-37). In contrast, pulsing DCs with KLH to provide unspecific help for CD8 ${ }^{+} \mathrm{T}$ cell induction $(29,38)$ in cohort 2 (patient \#31, \#33-53), significantly enhanced HLA-A2 tumor peptide-specific T cell responses over those induced by cocktail-matured DCs that were not KLH-pulsed (Figure 2B), particularly with respect to longevity $(P=0.0008)$. Immune responses to class I and II tumor peptides did not differ between tumor-bearing and tumor-free patients (Supplemental Figure 2A), between stage III and IV patients (Supplemental Figure 2B), or between patients with long or short survival (Supplemental Figure 2C). There was also no correlation between OS and KLH-specific responses (Supplemental Figure 8).

ELIspot assays were supplemented by extended analyses at apheresis time points (i.e., prevaccination and after 4 vaccinations). Ex vivo frequencies and phenotype of $\mathrm{T}$ cells in blood were evaluated by ex vivo class I tetramer staining in all but 3 patients. The highest average frequencies after vaccination were 
A

ELlspot responses - tumor peptide specific responses
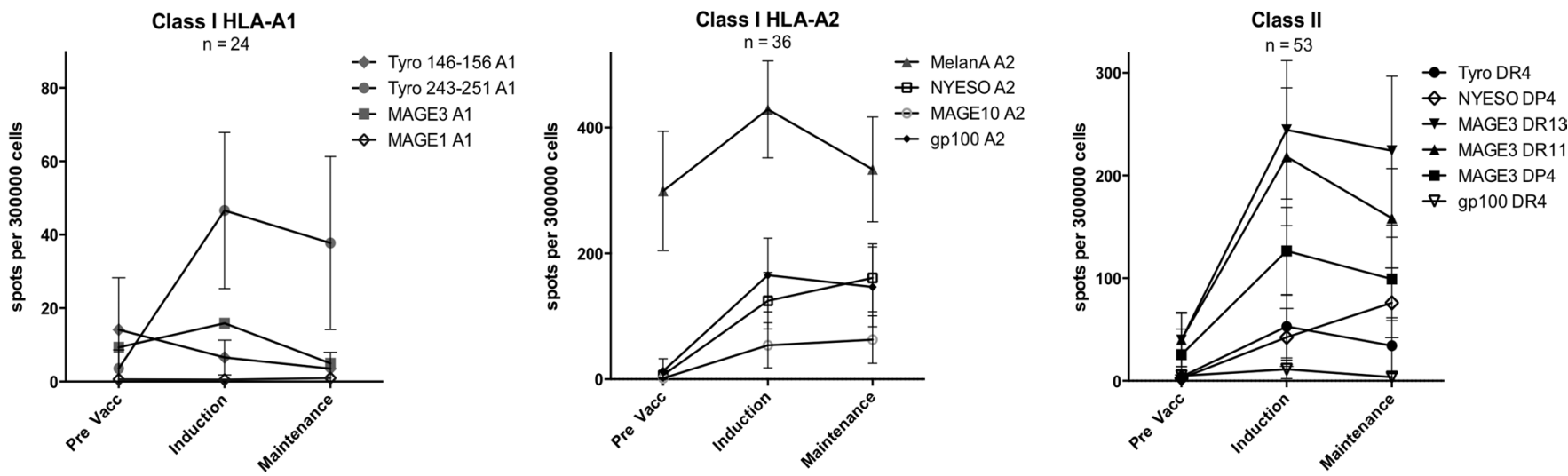

B

ELlspot responses - cohort $1(\bullet$ and $\bullet)$ vs cohort $2(\nabla)$

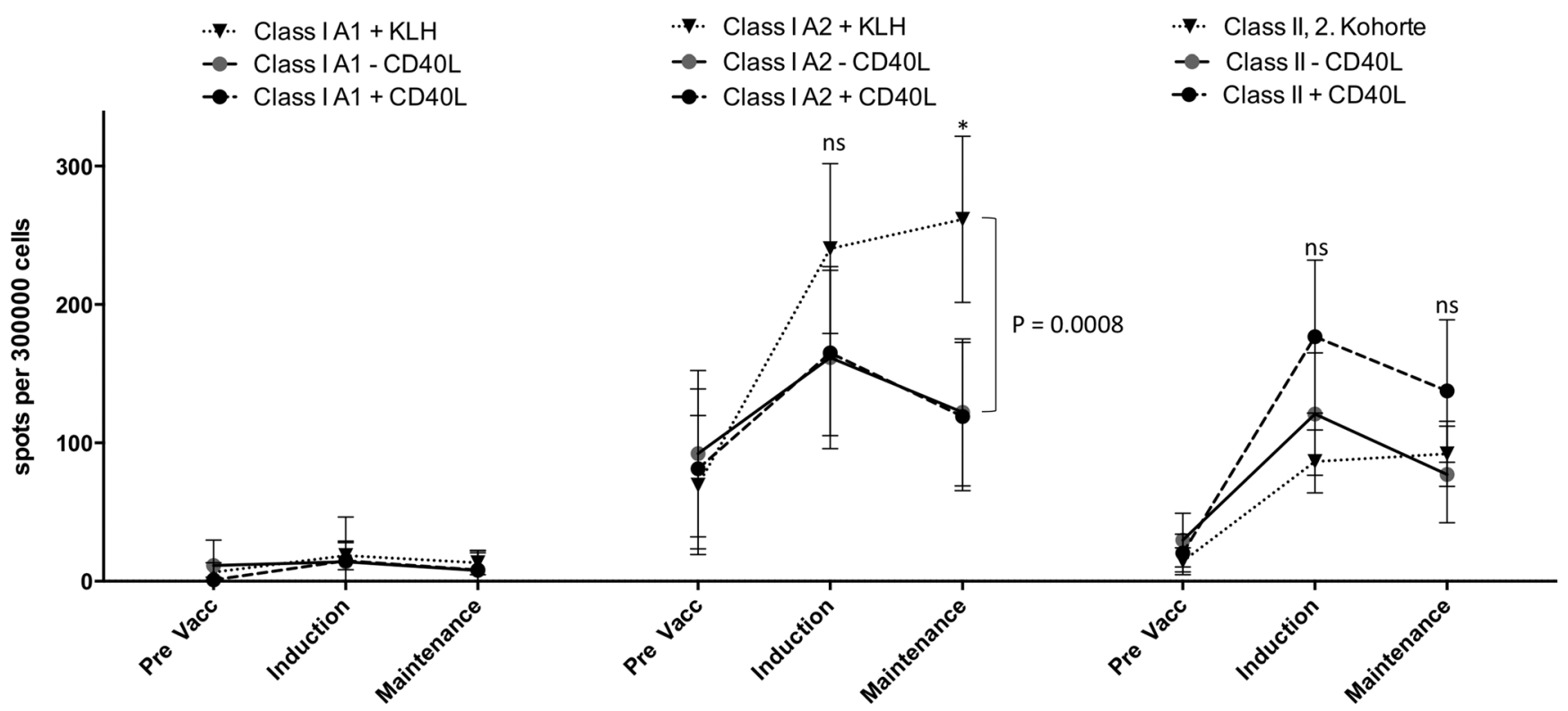

Figure 2. Overview of immune responses. Immune responses of all patients to vaccine peptides before and during induction and maintenance phase of DC vaccination as measured by in vitro-stimulated IFN $\gamma$ ELIspot are shown. Data from several time points are combined (1 or 2 before and 2-6 time points for induction and maintenance phase, respectively). Plots show the means with whiskers approximating the $95 \% \mathrm{Cl}$. (A) Cumulated responses of all patients to the different tumor peptides used. (B) Immune responses in each cohort combined for HLA-A1-, HLA-A2-, and the class II-restricted vaccine peptides. For comparison of KLH-DC- and CD4OL-DC-induced responses, an unpaired 2-tailed $t$ test with the assumption of inconsistent SD among samples and a false discovery rate approach of $1 \%$ was used.

observed for Melan-A and NY-ESO- 1 and reached up to $0.3 \%$ and $0.15 \%$ of $\mathrm{CD} 8^{+} \mathrm{T}$ cells, respectively (Figure 3A). The hierarchy of the class I-restricted tumor peptides with respect to the peak of postvaccination levels was similar to that observed by ivsELIspot (Figure 2). Of note, the CD45RA expression of vaccine-specific - but not total or control CMV-specific T cells - decreased, suggesting specific priming of naive $\mathrm{T}$ cells and differentiation into memory or effector memory cells (Figure 3B). The frequency of these nonvaccine specific T cells (Supplemental Figure 9) did not change during vaccination.

Ex vivo class II tetramer staining was performed for the Tyrosinase-HLA-DR4 epitope in 3 HLA-DR4 ${ }^{+}$ patients over time; it confirmed ELIspot findings and, in addition, proved that the vaccine-specific $\mathrm{CD} 4^{+}$ helper T cells were FOXP3-negative and CD127-positive, and thus did not constitute Tregs but rather vaccineinduced effector $\mathrm{T}$ cells (Figure $3 \mathrm{C}$ ). In congruence with this observation is the finding that total frequencies and proportions of proliferating Tregs did not significantly change upon vaccination (Supplemental Figure 3). 
A
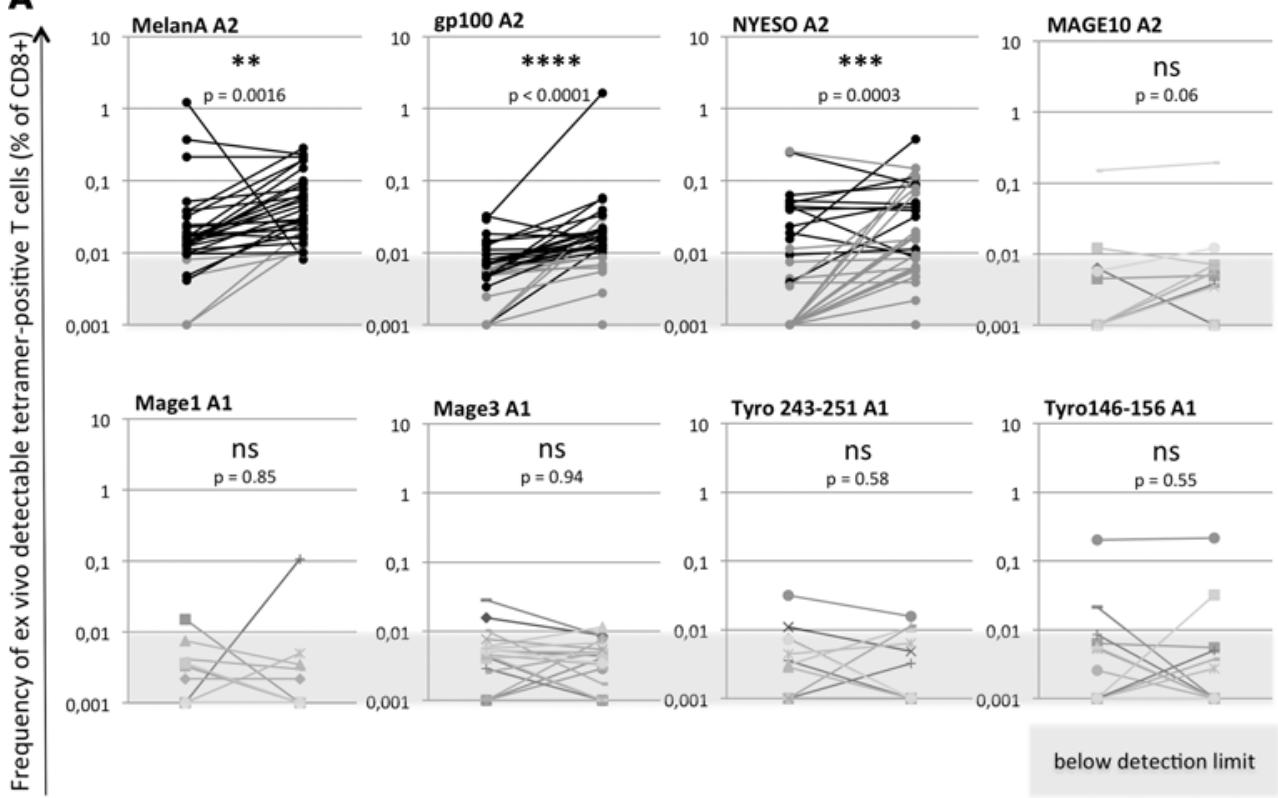

B

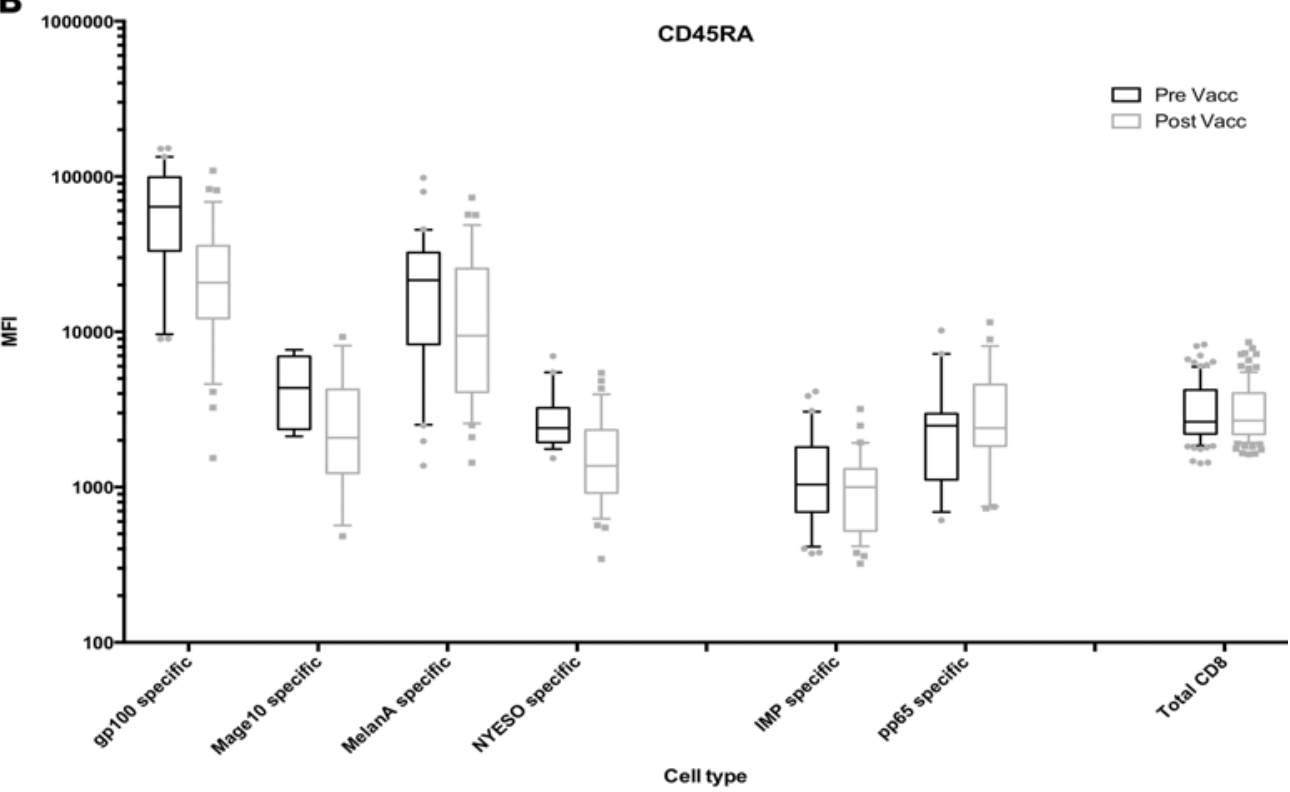

C

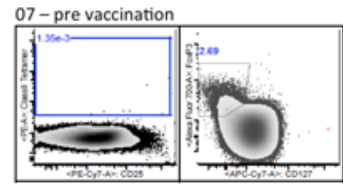

07 - Leu2 (after vacc \#4)

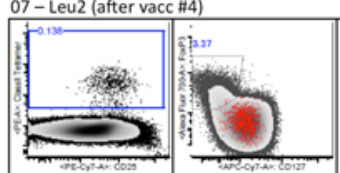

07 - Leu3 (after vacc \#7)

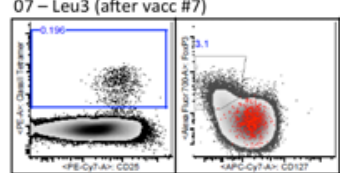

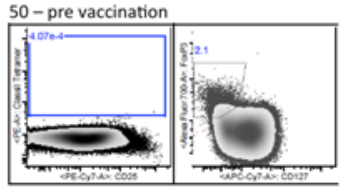

50 - Leu2 (after vacc \#4)

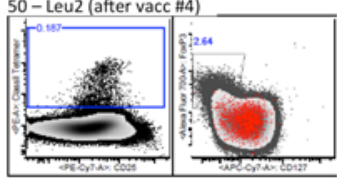

50- Leu3 (after vacc \#7)

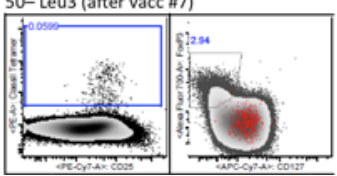

62 - pre vaccination

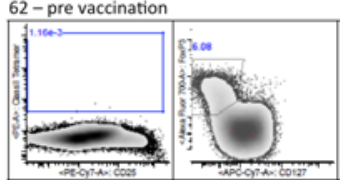

62 - Leu2 (after vacc \#4)

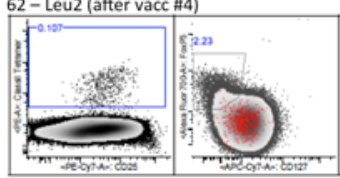

62 - Leu3 (after vacc \#7)

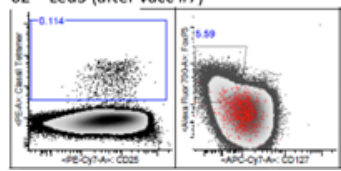

Figure 3. Ex vivo cell analysis. (A) Ex vivo multiplexed tetramer stainings were performed in 49 patients. Ex vivo-detectable frequencies of vaccine-specific $\mathrm{CD} 8^{+} T$ cells are shown as $\%$ of total CD8 ${ }^{+} T$ cells. Note the logarithmic scaling. Values below the detection limit are shown with gray background. Black lines and dots represent patients with a response that met the response definition criteria (at least 10 events in the tetramer gate and at least $0.01 \%$ of $\mathrm{CD}^{+} \mathrm{T}$ cells). For statistical analysis, a ratio-based 2-tailed paired $t$ test with $95 \%$ confidence level was used. (B) Downregulation of CD45RA in ex vivo-detectable vaccine-specific $T$ cells in comparison with virus-specific and total $\mathrm{CD} 8^{+} \mathrm{T}$ cells each before and after vaccination is shown as box plots of CD45RA mean fluorescence intensity (MFI) means with $95 \% \mathrm{Cl}$. (C) Ex vivo class Il tetramer staining (Tyro-DR4) is shown for patients 07,50 , and 62 at leukapheresis time points in combination with staining for $\mathrm{FoxP3}$ and CD127. In the right panel, total CD4+ $T$ cells are overlayed with tetramerpositive $\mathrm{CD} 4^{+} \mathrm{T}$ cells (red).

Also, the frequencies of myeloidderived suppressor cells (MDSC) remained unchanged (Supplemental Figure 3).

In addition to ex vivo tetramer stainings, classical mixed lymphocyte cultures (MLPC) in limiting dilution conditions were also performed to determine the minimal precursor frequencies, proliferative potential, polyfunctionality, and lytic capacity of vaccine-induced $\mathrm{T}$ cells. In agreement with the tetramer and ELIspot data, the $\mathrm{CD}^{+} \mathrm{T}$ cell precursors specific for HLA-A2-restricted tumor peptides increased significantly upon vaccination, even in patients with low or even undetectable prevaccination levels, demonstrating the capacity of the cocktail-matured DC vaccine to induce strong expansion of antigen-specific $\mathrm{CD}^{+} \mathrm{T}$ cells in vivo (Supplemental Figure 4A). Some T cell clones showed polyfunctionality with similar production of 3 or even 4 
cytokines produced at the same time (Supplemental Figure 4B). In addition, T cell clones were able to lyse target cell-loaded peptides at $10 \mu \mathrm{M}$ but also in many cases at $10 \mathrm{nM}$, indicating a high functional avidity (Supplemental Figure 4C). This is in line with the lysis of autologous or HLA-matched melanoma cell lines, as shown in Supplemental Figure 4D.

Luminex cytokine assay of culture supernatants of PBMC stimulated with a mixture of the class I and II tumor peptides versus control (Supplemental Figure 5) revealed that IL-4, IL-5, and IL-17 were low or virtually absent, while IFN $\gamma, \mathrm{GM}-\mathrm{CSF}, \mathrm{TNF} \alpha$, and - surprisingly - IL-13 were produced in most of the vaccinated patients. Compared with maximal stimulation of PBMC with anti-CD3/CD28 beads, the PBMC stimulated by vaccine peptides - both the class I- and class II-restricted peptides - produced significantly less IL-2, indicating their effector/effector memory phenotype.

Figure 4 is a summary of all T cell responses detected by ELIspot, MLPC, and/or tetramer staining, as well as the number of tumor-specific epitopes detected before and after vaccination. Several aspects are of interest. First, it is obvious that the ivsELIspot was the most sensitive assay, followed by the MLPC assay. Both showed good correlation, which was expected since both assays investigate in vitro-stimulated cells. Overall, there was, however, also a decent correlation to ex vivo-detected T cells. Particularly interesting was the total number of positive epitopes (criteria for judging positive epitopes are described in the legend to Figure 4) and the number of de novo tumor-peptide-specific responses upon vaccination, considering that 4 HLA-A1- and 4 HLA-A2-restricted and 6 HLA-class II-restricted tumor peptides were used in each patient. It was remarkable to see that, on average, 5.72 (i.e., 95\%) of the maximum possible 6 reactions to the class II epitopes were found positive after vaccination using IFN $\gamma$ production as readout, and that 4.36 (i.e., a remarkable 73\%) were not detectable before vaccination and were thus probably induced de novo (i.e., primed upon vaccination). In the case of HLA-A2 patients, reactivity to the 4 class I tumor peptide epitopes was also found in the majority of cases after vaccination (on average, 3.69 of 4 possible, i.e., 92\%), but only 1.77 (44\%) emerged de novo. In HLA-A1 patients, a much lower, 46\% of positive epitopes (1.83 of possible 4), was found after vaccination. This was no surprise, given the lower immunogenicity of HLA-A1 peptides observed in all quantitative assays. Interestingly, however, judging only the number of de novo $\mathrm{T}$ cell responses, the HLA-A1 epitopes were less inferior, in that $32 \%$ (1.29 out 4 possible) emerged de novo.

Distribution and intensity of tumor infiltrating $T$ cells. A T cell-rich micromilieu seems a favorable prognostic marker in tumors, depending on the immune contexture $(39,40)$. In cutaneous melanoma, the prognostic value of tumor infiltrating lymphocytes in the primary tumor, as well as lymph node metastases, has been suggested 20 years ago $(41,42)$ and has recently been confirmed and expanded $(43-45)$. Transcriptome studies had then suggested that a $\mathrm{T}$ cell-inflamed milieu in pretreatment melanoma metastases is associated with favorable clinical outcome to melanoma vaccines (46) and other immunotherapies such as anti-CTLA-4 $(47,48)$. To gather information on the tumor microenvironment in the current trial, we studied the distribution and intensity of tumor infiltrating $\mathrm{CD}^{+} \mathrm{T}$ cells, in order to determine a melanoma immunoscore. To this end, we used a semiquantitative approach (lymphocyte or L-Score) that has recently been shown in melanoma to strikingly correlate with the transcriptomic immune subclass and also with prognosis (favorable for score 3-6, unfavorable score 0-2) (45). We were able to analyze prevaccination metastases in 17 patients and postvaccination metastases in 17 patients with corresponding pre- and postvaccination metastases in only 7 patients. The respective quantitative lymphocyte scores (range 0-6, see Methods) of pre- or postvaccination metastases are listed in Supplemental Table 2. The data set illustrates that a favorable higher lymphocyte score was found only in $24 \%$ (4 of 17) available prevaccination metastases, indicating that our patient population was not enriched for an immunologically highly favorable subset, considering that overall $40 \%$ of melanoma metastases are reported to be T cell-rich in large data sets (45, 49). After the vaccinations, high lymphocyte score metastases occurred in more patients (8 of 17) as one would expect from a vaccine effect, but the numbers of accessible metastases were too small for a meaningful statistical evaluation regarding correlation with OS.

Safety and tolerability (toxicity). No major (> grade 2) toxicity was observed in any patient. We noted, however, as in previous trials, 2 typical side effects, namely a transient mild rise in body temperature (only 3 patients developed temperatures over $38^{\circ} \mathrm{C}$ to maximally $39^{\circ} \mathrm{C}$ ) sometimes accompanied by constitutional symptoms, and vaccine injection site reactions in $92 \%$ of patients. Both side effects typically showed up for the first time after the second to fourth vaccine administration, with a delay of 6-16 hours after DC injection. Seven patients (\#04, \#15, \#21, \#29, \#36, \#45, and \#60) developed a grade 2 (based on common toxicity criteria version 2.0 [CTCv2.0]) rash (for examples, see Figure 5) with onset 2-4 days after vaccination and 


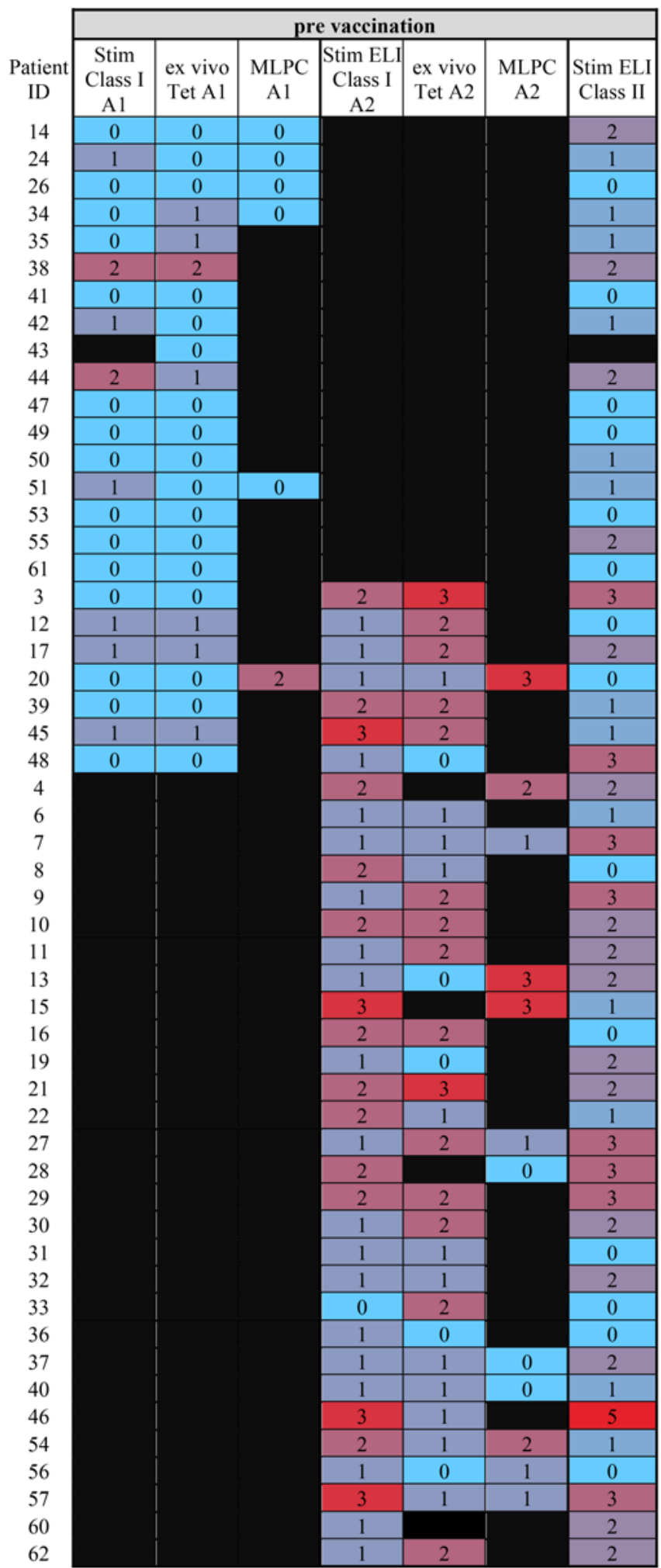

intermediate response

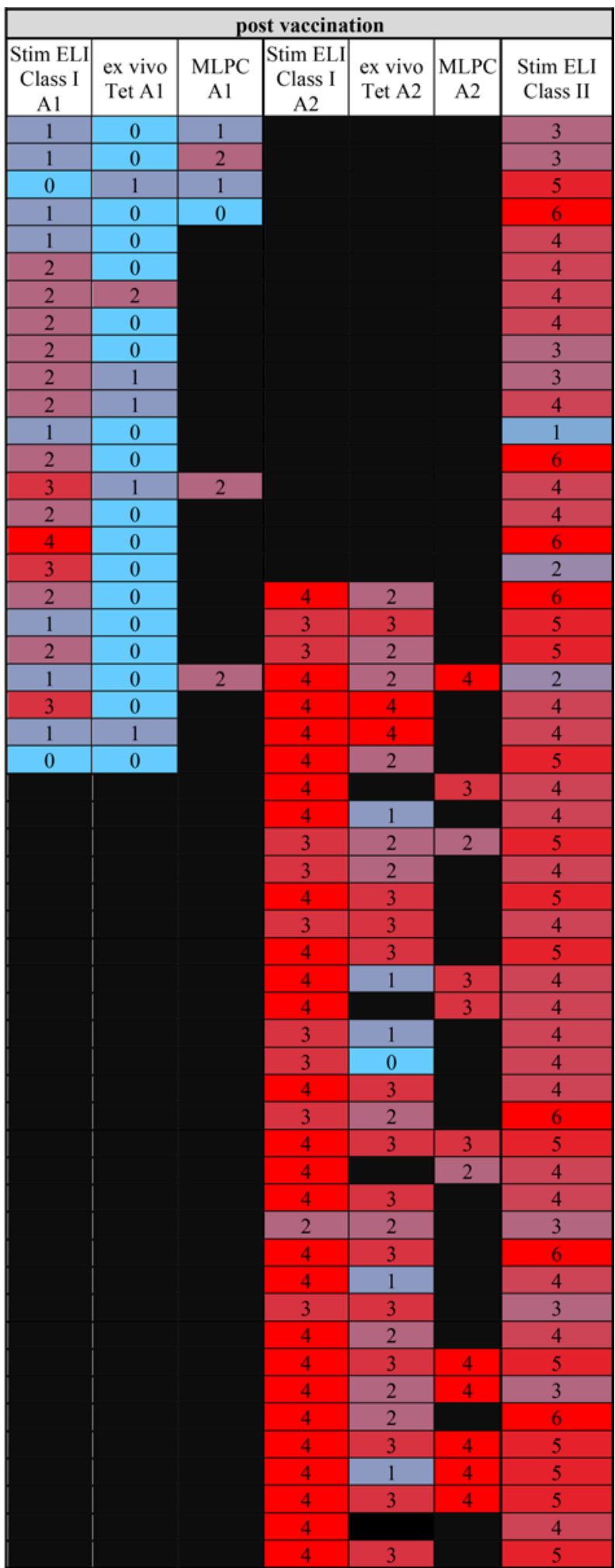

no response not done/ not applicable

Figure 4. Overview and breadth of DC-vaccination-induced immune responses. Shown are the numbers of positive immune responses (possible maximum of 4 responses to class I and 6 to class II tumor peptides ) detected by the different methods and according to the following response criteria: ELIspot, at least 2.5-fold above background and at least 10 spots after substraction of background at any time point; ex vivo tetramer, at least $0.01 \%$ of tetramer-positve cells among vital CD8 ${ }^{+} \mathrm{T}$ cells and at least 10 events in the tetramer gate; and MLPC, at least 1 well of the limiting dilution assay wells needs to be present with sufficient tetramer-positive cells. 


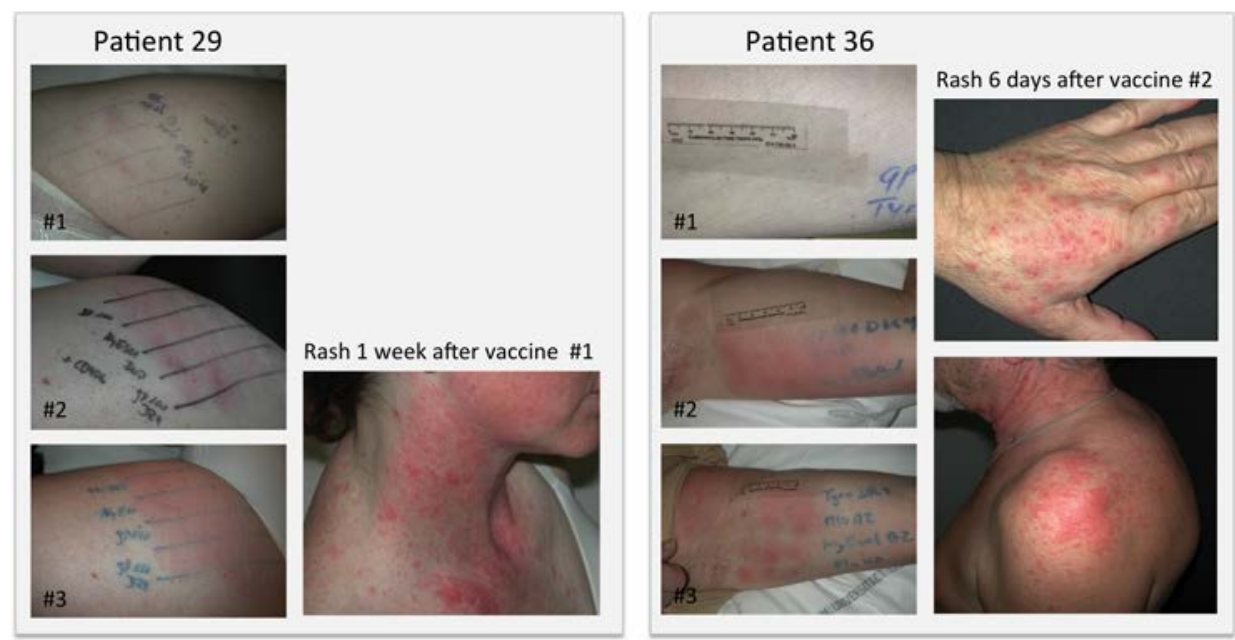

Figure 5. Local vaccine reaction and rash. Massive DTH reaction with formation of very large indurated areas (up to $20 \mathrm{~cm}$ ) at the intradermal injection sites (photos of 1 limb only); simultaneous development of a rash (face, trunk, arms in patient 29 and in patient 36 ). lasting for several days. We had never observed the occurrence of a rash in our previous DC vaccine trials with identical DC generation protocols and equal GMP peptides. The only discernible difference to previous trials was that we had substituted autologous serum with pharmaceutical-grade HSA as a component of the cryopreservation cocktail, since HSA is widely used in cryopreservation media as a substitute. We performed intracutaneous allergy tests and found that delayed hypersensitivity was detectable to various brands of HSA tested, including the one used in the trial, but not to autologous serum or the nonprotein compounds contained in HSA preparations. Immediate hypersensitivity was not observed. As a consequence, in patients who had

developed these side effects, another apheresis was performed in order to produce DCs cryopreserved without HSA. Vaccinations with these DCs did not produce the side effects described above. All other DC vaccinations for subsequent patients (from \#50 onwards) were generated without use of HSA for the cryopreservation medium, and as expected, no further rashes occurred. These observations suggested to us that HSA should be avoided in the context of potent DC vaccines, as immune-mediated side effects may occur perhaps because virus-inactivated commercial HSA preparations get differentially processed and generate epitopes to which central tolerance has not been induced in the thymus.

Clinical impact and correlation of prolonged OS to immune parameters. We did not observe regressions according to WHO criteria (defined for evaluation of tumor response in the clinical trial protocol) - that is, a complete or partial regression of all metastases — but as in our previous DC vaccination trials, we observed a slow regression and eventual complete disappearance of individual metastases (Supplemental Figure 6A). In patient \#06, excised pulmonary metastases proved, in fact, to be granulomas with few, if any, tumor cells left (Supplemental Figure 6B). The most striking observation arising from the trial was that many patients, even after initial progression, stabilized. Thus, currently after a minimum of a 12-year followup, 19\% of patients suffering from metastatic melanoma with measurable disease are still alive (Figure 6). None of these patients had received any of the new kinase or checkpoint inhibitor therapies except patient \#36, who received 2 ipilimumab infusions $(3 \mathrm{mg} / \mathrm{kg}$ ) 7 years after the start of the trial. Long-term survivors resulted in the formation of a plateau at the end of the Kaplan-Meier plots (Figure 6), as first observed with anti-CTLA-4 immunotherapy and now considered typical for successful cancer immunotherapies $(50,51)$. OS of the smaller group of 21 tumor-free patients that was included (7 resected stage IV and 14 resected stage III patients) also compared very favorably with what others have observed upon vaccination with mRNA-transfected DCs combined with IFN 2 b (52), DC vaccines alone (53), or nivolumab combined with a multipeptide vaccine (54); it even compared well (with $75 \%$ OS at 5 years) with the recently published OS data of adjuvant ipilimumab treatment of resected stage III patients (55).

Statistical analyses revealed, however, that there was no significant correlation between long OS in metastatic patients and the strength of vaccine-specific immune response (Supplemental Figure 2C) measured in the blood in the induction or maintenance phase. There was also no obvious correlation with the number of recognized tumor epitopes (that is, the breadth of vaccine-specific responses) even if one took into account solely the de novo responses. A lack of such a correlation was probably also due to the fact that, in the majority of patients (74\%) - including HLA-A1 patients - at least 4 newly recognized epitopes emerged after vaccination, and in all but 2 patients (i.e. ,96\%), immunity to at least 2 new vaccine epitopes was induced.

Looking for other immune-dependent correlates, we found a statistically significant correlation between OS and the intensity of vaccine injection site reactions (Figure 7A). As a truly unanticipated finding in 2011 (see Discussion), a significant correlation $(P=0.0018)$ between the emergence of eosinophilia $(>250 / 100$ 


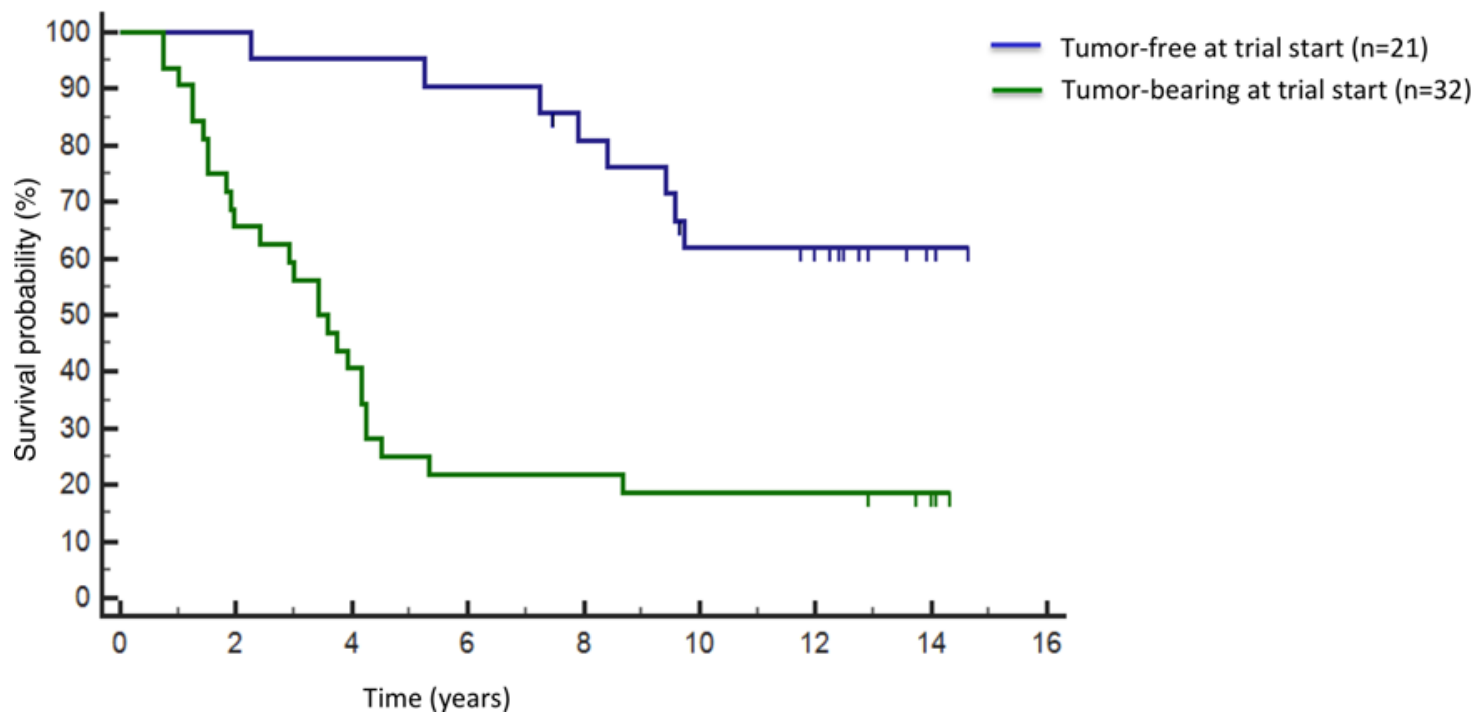

Figure 6. Overall survival. Kaplan-Meier analysis of overall survival of fully evaluable metastatic (stage III + IV) melanoma patients (for characteristics, see Figure 4), either tumor-free or nonresectable. Two patients were censored after 87 and 117 months due to death unrelated to melanoma. None of the tumor-bearing patients still alive underwent therapy with kinase inhibitors. Only 1 patient underwent therapy with checkpoint blockade antibodies (patient 36 received 2 infusions of ipilimumab - 7 years after trial start in September 2011 - that had to be stopped due to autoimmune colitis. DC vaccination using a different method of tumor antigen loading, namely transfection with autologous tumor mRNA, was started in April 2012 and was kept up until recently).

$\mu 1)$ after vaccination and subsequent long-term survival emerged from the safety analysis (Figure 7B). Interestingly, this correlation was significant only for the tumor-bearing patients $(P=0.0018)$. Of note was also that absolute eosinophil counts before vaccination showed no correlation to later survival (data not shown). As one would expect, there was a significant correlation $(P=0.0019)$ between vaccine injection site reactions and eosinophilia (data not shown), underscoring their potential role as a useful predictive biomarker. The use of HSA in the vaccine did not correlate with survival or with eosinophilia and vaccine injection site reactions (data not shown).

\section{Discussion}

To our knowledge, this is the only DC vaccination trial that follows up each single patient for at least 10 years and also provides immune monitoring data for all patients. This allowed us to reveal significant correlations between long-term survival, indisputably the most important clinical efficacy parameter, and our analyses. In our current trial, we have confirmed our previous data, implicating that vaccinespecific $C D 8^{+} \mathrm{T}$ cells expanded by the current DC type are $\operatorname{IFN} \gamma$-producing and lytic $(24,33,56,57)$, cover a wide spectrum of TCR affinities including high-affinity CD8 ${ }^{+} \mathrm{T}$ cells capable of killing HLAmatched or autologous melanoma lines, and remain as memory $\mathrm{T}$ cells even at prolonged vaccination intervals. We now also demonstrated in accordance with recent reports (58) that the vaccine-specific $\mathrm{CD}^{+} \mathrm{T}$ cells were polyfunctional, a property considered relevant for antiviral (59-61) and antitumor efficacy (62-64).

The primary goal of this trial was to even improve the immunogenicity of our "standard" tumorpeptide-loaded, cocktail-matured, monocyte-derived DCs by testing the influence of different DC-licensing strategies. In the first cohort, additional exposure to bioactive trimeric soluble CD40L (also known as CD154) was used to elicit higher vaccine-specific responses based on the demonstrated enhanced in vitro activity of CD40 triggered monocyte-derived DCs $(27,28,34,35,37,65)$. The rationale was that triggering of CD40 on DCs is known to promote their maturation and activation, as well as their survival, and - more importantly in the context of our study - it has also been shown in seminal studies to license the DCs for optimal expansion and activation of CD8 $8^{+}$killer T cells ("license to kill") (29, 30). In the second cohort of patients, DCs were pulsed with $\mathrm{KLH}$ to provide unspecific help and licensing by $\mathrm{CD} 4^{+} \mathrm{T}$ cells in vivo.

Surprisingly, it turned out that the in vivo immunogenicity of DCs could not be enhanced by triggering CD40 on DCs by exposure to trimeric soluble CD40L before their adoptive transfer. This was not due to a 


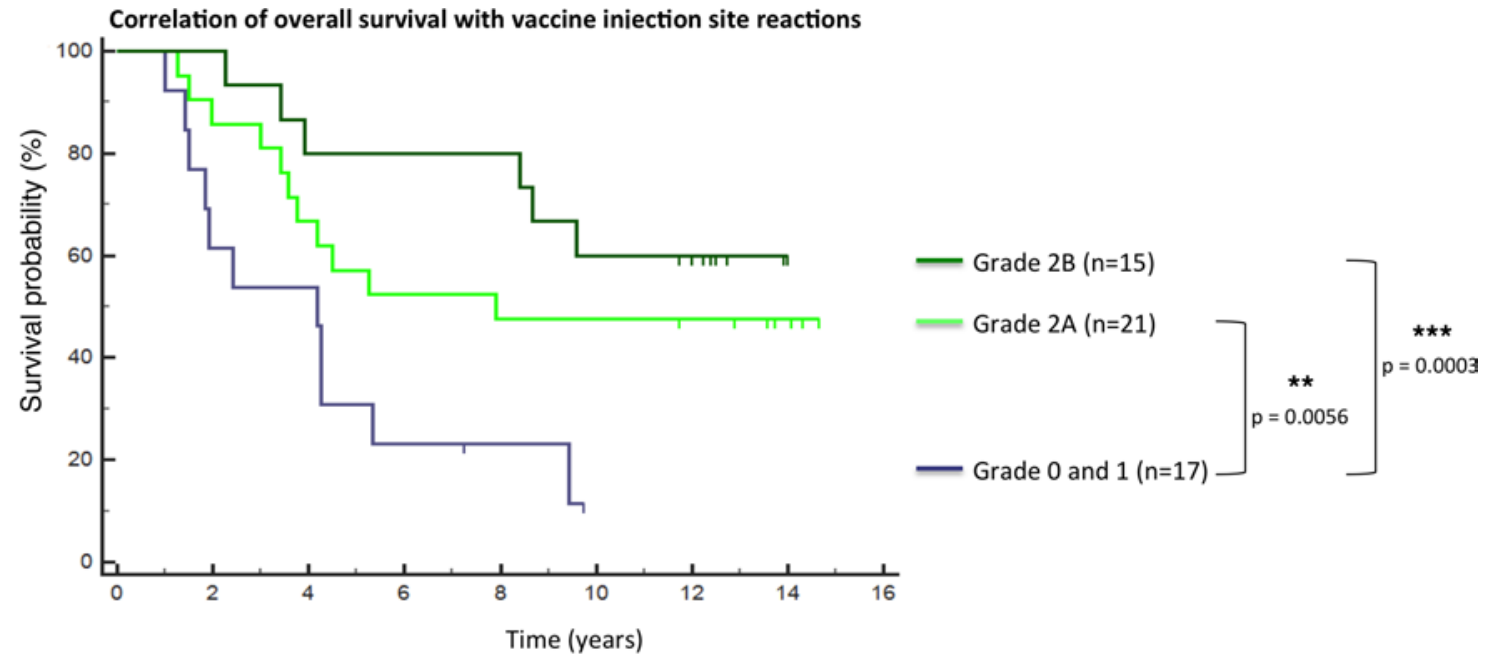

Maximal abs. eosinophil counts after vaccination (vacc \#2 - vacc \#10)

Correlation of overall survival with absolute eosinophil counts after vaccination

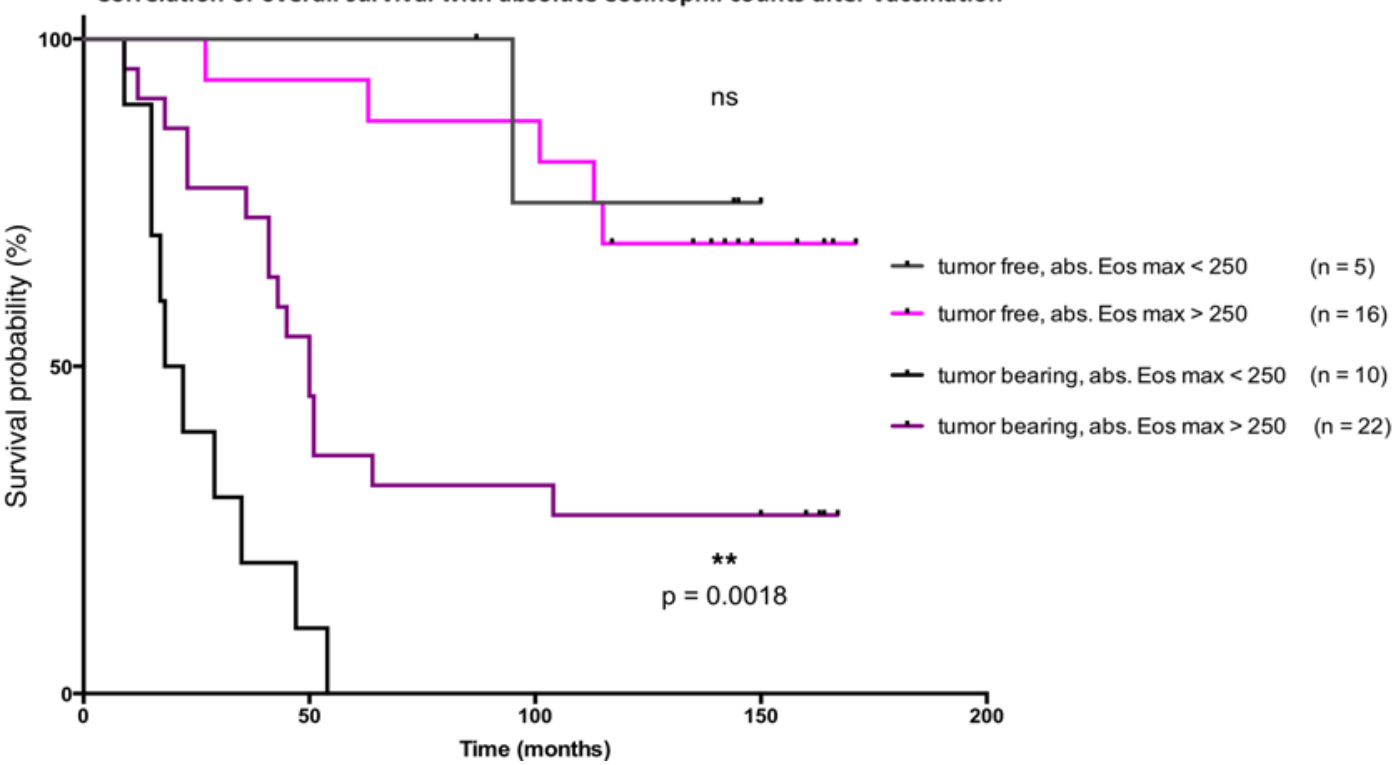

Figure 7. Correlation of overall survival with vaccine injection site reactions and with development of eosinophilia upon vaccination. (A) KaplanMeier analysis of overall survival according to differences in local reactions at vaccine injection sites. Reactions were either absent $(n=4)$, grade 1 (redness, $n=13$ ), or grade 2 (redness and edema, $n=36$ ) according to CTC criteria v 4.0. We subcategorized CTC grade 2 further in grade 2A (redness and induration) and grade $2 \mathrm{~B}$ (redness and induration surrounded by a white margin due to very strong edema). Two patients censored after 87 and 117 months due to death unrelated to melanoma. For comparison of curves, a log rank (Mantel-Cox) test was used. The stronger the DTH reaction, the better the survival. If grade $0+1$ were compared with grade 2 (i.e., grade $2 \mathrm{~A}$ and grade 2B pooled), the $P$ value was $P<0.0001$. (B) Kaplan-Meier analysis of overall survival according to maximal absolute eosinophil counts (count per $100 \mu \mathrm{l}$ of blood) after vaccination (time points from vacc \#2 until vacc \#10) in tumor-free and tumor-bearing patients. Two patients censored after 87 and 117 months due to death unrelated to melanoma death. For comparison of curves, a log rank (Mantel-Cox) test was used.

lack of bioactivity of CD40L, as it enhanced - as described (34) - in vitro survival of the DC vaccines. Since class I and II peptides were loaded in a randomized fashion onto different batches of DCs (to avoid licensing by help in cis from CD40L expressing $\mathrm{CD} 4^{+}$helper $\mathrm{T}$ cells activated by the DCs) within each patient, any bias from immune competence of patients or different immunogenicity of peptides did not account for the missing difference between CD40L-pulsed and CD40L-unpulsed DCs. In contrast to this failure of CD40 triggering, in the second cohort, unspecific help - induced via KLH-pulsing of DCs - significantly enhanced HLA class I-restricted responses, especially with respect to their longevity. This result prompted us to examine more closely the mechanism of T cell help to (monocyte-derived) DCs. It turned out that the signal delivered 
via CD40 is too transient (66). Based on these insights, we have recently established a protocol by transfecting mRNA coding for constitutively active mutants of IкB kinases (caIKKs) that better mimics prolonged activation (i.e., licensing) of DCs by helper T cells. This allows generation of highly immunogenic DCs that seem superior for $\mathrm{T}$ cell priming and memory generation (67), which we will test in a clinical trial.

Our observation of a favorable clinical outcome is not new per se, as putative prolonged os has been reported by others in subsets of their DC-vaccinated patients with nonresectable (68-81) or resected (52, $69,75,76)$ melanoma metastases. There is, however, no DC vaccination trial published with a predefined minimum 10-year followup of all patients, as reported here. Of course, patient numbers in our trial are small, and a biased selection of favorable patients might have occurred; however, our prolonged followup allowed us to detect a group of survivors that show long-lasting survival with a plateau forming at 3 years, similar to successful cancer immunotherapy in ipilimumab-treated metastatic melanoma patients $(50,51)$, and to find correlations with immune parameters. In 2011, we initially suspected this plateau of survivors forming in our DC-vaccinated patients, and at that year's Keystone Symposium "DCs and the Initiation of Adaptive Immunity (J7)" (http://www.keystonesymposia.org/11J7) also reported for the first time to our knowledge the remarkable observation that the presence of eosinophilia after the first $4 \mathrm{DC}$ vaccinations strongly correlated with and even predicted long survival. Most notably, a correlation of eosinophilia upon DC vaccination was then also observed in the Provenge phase III vaccination trials (82) in metastatic castration-resistant prostate cancer and, more recently, has also been reported by several groups in ipilimumab-treated patients, where an early increase in eosinophil count was also associated with an improved clinical outcome (83-85). These observations suggested that this might be a relevant phenomenon with an underlying common mechanism for improved survival. Indeed, Hämmerling et al. have recently reported, in a mouse melanoma model, that activated eosinophils were essential for tumor rejection not by a direct tumoricidal effect (although it has been reported in mice and humans in vitro; refs. 86-91), but by enhancing the infiltration of $\mathrm{CD}^{+} \mathrm{T}$ cells via secretion of chemoattractants and normalizing tumor vessels, thus providing a mechanistic link to understanding why an increased eosinophil count may herald a better outcome (92). Of note is that tumor eosinophil infiltration has been described as favorable (e.g., in colon cancer) (93) or unfavorable (94). Its occurrence and relevance in human melanoma remain to be studied in more detail, but it is striking that Soiffer et al. observed both blood eosinophilia and degranulating eosinophils in regressing melanoma metastases upon vaccination with GM-CSF-secreting autologous melanoma cells (95). Furthermore, it will be important to elucidate how the observed transient increase in blood eosinophilia upon our DC vaccination (as well as ipilimumab therapy) is mediated. The simplest explanation would be IL-2 secreted by vaccine-specific T cells mediates the increase, as IL-2 upon injection even at low doses is known to cause blood eosinophilia (90). Another possibility is that cytokines such as GM-CSF preferentially produced by DC vaccine-induced tumor-specific T cells (see results) play a role similar to what Soiffer et al. had observed (95).

In addition to eosinophilia, we observed a second factor correlating with OS: strong vaccine injection site reactions, which also significantly correlated with eosinophilia. Indeed, in such DC vaccine reaction sites beside antigen-specific T cells $(96,97)$, an infiltration with eosinophils was also found (98), meaning that the vaccine injection site reactions should constitute an ideal and accessible model to study the role of eosinophils in $\mathrm{T}$ cell migration and mechanistic issues also in man. A trend for the correlation between local vaccine reactions and clinical benefit has been reported in various DC vaccine trials previously, but results were inconsistent $(99,100)$. Boudewijns et al. in a recent retrospective analysis of a larger group of heterogeneously DC-vaccinated patients observed, for example, that there is a correlation between injection site reactions and both the presence of antigen-specific $\mathrm{CD}^{+} \mathrm{T}$ and survival (101). In another study, they found, however, that tumor-specific $\mathrm{CD} 8^{+} \mathrm{T}$ cells isolated from delayed-type hypersensitivity (DTH) sites after intradermal challenge with antigen-loaded DCs (so-called skin-test infiltrating lymphocytes) predicted and correlated with the clinical outcome, while DTH induration was neither associated with OS nor tumorantigen-specific $\mathrm{CD}^{+} \mathrm{T}$ cell responses (102). We hypothesize that, in our case, the emigration potential and local proliferation of tumor-specific $\mathrm{T}$ cells at the vaccine site was so prominent that the clinical readout of a strong vaccine injection site reaction alone revealed a statistically significant correlation with survival. Both the strong local vaccine reaction, as well as the clinical benefit, may be due to the emigration potential of fit vaccine-induced T cells. Data obtained by employing platforms for the parallel detection and high-dimensional characterization of many different $\mathrm{T}$ cell specificities indicate that it will be rewarding in future trials to investigate which DC types result in better tissue immunity $(103,104)$. 
We, of course, asked ourselves why there is no significant correlation between long survival and induced tumor-specific immune responses as measured in the blood. The lack of such a significant correlation is, however, not really surprising. Overall, in vaccine trials, such correlations have been variable, and sometimes, only the number of recognized epitopes has shown correlation with survival in nonrandomized trials. More important is the finding that $\mathrm{T}$ cell responses emerging in the tumor microenvironment to nonvaccine antigens are more relevant (105-107). It was shown that even weak and barely detectable vaccine-specific T cells in blood, provided they are of high quality, can serve as a "spark" in non- or only moderately suppressive tumor microenvironments and promote local inflammation. This then results in epitope spreading to other antigens, including mutated ones, which are currently considered critical rejection antigens. Interestingly, the standard DC vaccine as used in our study (i.e., cocktail-matured monocytederived DCs), but loaded with dying autologous tumor cells in 2 small trials $(70,71)$, also resulted in longterm survival and, as recently shown in neo-epitope-specific T cells (81), underscoring the immunogenicity of this simple yet highly standardized nontoxic DC vaccine.

In view of this documented immunogenicity of the standard DC vaccine, and in view of evidence for prolonged survival in several trials, the question arises as to why a previous randomized trial comparing first-line dacarbazine chemotherapy (52 patients) to vaccination with the same DC type (41 evaluable patients) in a comparable patient population did not produce clinical benefit and was, therefore, prematurely terminated (108). There are several plausible explanations for this difference. One could be that only half as many class II-restricted tumor peptides inducing $\mathrm{CD}^{+} \mathrm{T}$ cell responses were used. Tumor-peptide-specific helper $\mathrm{T}$ cells - apart from their established role to promote generation, infiltration, and local activity of $\mathrm{CD}^{+} \mathrm{T}$ cells - are now known to display antiangiogenic and direct tumor-destructive properties, and to induce tumor cell senescence or even, alone, mediate cancer control (109-114). Noteworthy, the "spark" mentioned above, leading to antigen spreading, is probably cytokine dependent. Therefore, $\mathrm{CD}^{+} \mathrm{T}$ cells, which produce larger amounts of cytokines than $\mathrm{CD} 8^{+} \mathrm{T}$ cells, should be very good inducers of this process. Another aspect is that the DC vaccine was delivered s.c. in the failed trial, a route now considered inferior to the intradermal injection of small DC numbers at multiple sites as used in the current trial. We believe, however, that the overall low quality of the DC vaccine (due to a suboptimal maturation cocktail) and the significant underdosing (a mean of 2.8 million DCs per antigen instead of an intended 4 million DCs per class I peptide were administered in contrast to 10 million in the current trial) played an even more decisive role in the failed trial, as the vaccine simply could not achieve the required immunogenicity. Indeed, we found a several-fold lower induction of vaccine-specific $\mathrm{T}$ cell responses when we recently studied samples of some patients of the halted phase III trial, in which immunomonitoring had not been planned.

We would also like to emphasize that only in the current trial, we designed and followed a strategy that forestalled later paradigm changes, in that we did not stop DC vaccination if progression was slow or moderate. In addition, we also performed a combination approach in that residual or progressing metastases, if accessible, were surgically removed or irradiated, which should have improved anticancer efficacy (115). We hypothesize that the prolonged vaccination was also important in providing new waves of new tumor-specific $\mathrm{T}$ cells that might have ignited antigen spreading and, by a still unknown pathway, resulted in transient eosinophilia, which is remarkably also occurring in ipilimumab-treated long-surviving patients as discussed above. We believe that our tactic significantly contributed to achieving long-term survival upon DC vaccination without major side effects. A pilot investigation of cryopreserved prevaccination metastases available in some of the patients had previously shown us that a $\mathrm{T}$ cell-inflamed transcriptome signature was associated with survival to our DC vaccine (116). We have now extended these analyses on more patients by determining a recently described lymphocyte score on paraffin sections (45). Importantly, the respective data show that our patient population was not enriched and, thus, biased for the $\mathrm{T}$ cell-rich subgroup. Interestingly, upon vaccination, an increase in the lymphocyte score occurred, which can be seen as possible vaccine effect.

In conclusion, the melanoma DC vaccination trial described here is special in that it was performed before the modern checkpoint and kinase inhibitors became available, it included serial immunomonitoring in all patients, and it had an unprecedented long-term followup of at least 10 years (currently 12 years) that presented a group of long-term survivors comparable with ipilimumab-treated patients. The long-term survivors were predicted by the emergence of strong vaccine-specific vaccine injection site reactions and the presence of eosinophilia after the first round of DC vaccination. In contrast to what in vitro studies had projected, CD40L could not further enhance the immunogenicity of the monocyte-derived DCs matured 
by the "standard" inflammatory cocktail. The immunogenicity and safety profile of this nontoxic DC vaccine is now firmly substantiated, and it seems an ideal combination partner for other therapies such as checkpoint blockade, particularly if electroporated with mRNA for antigen loading to circumvent restrictions imposed by HLA-restriction of peptides $(117,118)$. Several other testable hypotheses have emerged from this trial that could help to optimize cancer vaccines.

\section{Methods}

All Methods are described in brief. A more detailed description of all methods, including detailed inclusion and exclusion criteria, information on the production of vaccines, and reporting of methods and obtained data on immune responses in line with Minimal Information About T cell Assays (MIATA; www.miataproject.org) guidelines (119) is available as Supplemental Methods.

Trial design. The study was designed as a monocentric, open-label phase I/II DC vaccination trial (NCT00053391), to test the immunogenicity of cocktail-matured, peptide-loaded, monocyte-derived DCs with or without soluble CD40L as additional maturation stimulus (primary objective). Additional endpoints were to evaluate toxicity (coprimary endpoint) and clinical impact (secondary objective) in advanced stage IV or stage III cutaneous melanoma patients (metastatic or tumor-free, yet at high risk of relapse). Most important inclusion criteria were a life expectancy of $\geq 4$ months and expression of HLA-A*0201 and/or $-A^{*} 01$ to allow for loading with peptides restricted by these haplotypes. The most important exclusion criteria were active metastases of the brain. Chemo-/immune- and/or radiotherapy were to be stopped $\geq 4$ weeks preceding the first vaccination, and concurrent treatment was not permitted. However, excision or radiotherapy of select metastases was possible. Proof of tumor antigen/gene expression in metastases was not required for inclusion, as only frequently expressed antigens were chosen for the vaccine. The trial was started following approval by the local IRB (Universitär Hospital Erlangen, Friedrich-AlexanderUniversity Erlangen-Nuremberg (FAU), Erlangen, Germany.) and the federal regulatory authorities (Paul Ehrlich Institute, Langen, Germany) and conducted according to good clinical practice (GCP) criteria. All patients signed an informed consent, and a 10-year followup was planned. The study scheme is shown in Figure 1. The first 4 DC vaccinations (initiation phase) were administered at increasing intervals (2, 4, and 4 weeks, followed by a first evaluation one month later). Subsequently, another 6 vaccinations (maintenance phase) were scheduled over a total period of 2 years of vaccination with increasing time intervals ranging from 2-6 months. DC vaccinations were maintained according to the trial protocol if only slight progression occurred. Surviving patients had the option of receiving further vaccinations in 6-month intervals beyond the end of the trial (for a total number of vaccinations, see Supplemental Table 1).

Generation and administration of DCs. Standardized mature, monocyte-derived DCs were generated from apheresis as described previously (22). In brief, immature monocyte-derived DCs were exposed for 24 hours to a defined standard inflammatory cocktail for maturation (consisting of TNF $\alpha$, IL-1 $\beta$, IL- 6 , and $\left.\mathrm{PGE}_{2}\right)(120,121)$ and then loaded with 4 HLA-A1- and/or 4 HLA-A2.1-restricted tumor peptides (see Supplemental Table 3). Each class I peptide was loaded onto a separate batch of 12 million later cryopreserved DCs to avoid competition for the same HLA molecule (for peptides, loading and injection scheme see Figure 1). In addition, we also used 6 HLA class II tumor peptides restricted by HLA-DP4, -DR4, -DR11, and -DR13 (see Supplemental Table 3). Considering the promiscuous binding of class II peptides, they were loaded on DCs independent of the HLA type of the patients. For each vaccination, a total of 72 million tumor-peptide-loaded DCs were administered.

In the first cohort $(n=31)$, half of the mature DCs of each patient were additionally pulsed with recombinant, soluble trimeric CD40L. The 2 DC populations were administered into the deep dermis at the right versus left thigh (or, in the case of lymphadenectomy, at the upper arms). As a control for extent and quality of helper $\mathrm{T}$ cell priming and the general competence of the patient's immune system, once at onset, a single injection of only 4 million DCs pulsed with $\mathrm{KLH}$ protein $(10 \mu \mathrm{g} / \mathrm{ml})$ during maturation (to allow processing into HLA class II molecules) was given. Furthermore, for the initial 4 vaccinations, separate batches of influenza class I peptide-loaded DCs were included (12 million DCs as in the case of the tumor peptides) as a control.

For cohort $2(n=31)$, CD40L pulsing of half of the DCs was omitted, and instead, all DCs to be loaded with class I peptides at the mature stage were exposed during maturation to KLH protein (at a reduced concentration of $2 \mu \mathrm{g} / \mathrm{ml}$ ) to provide unspecific help for CTL induction (Figure 4). DCs to be loaded with class II peptides were, of course, not exposed to KLH. 
PBMC sample collection, preparation, and storage. PBMC were isolated from blood or aphereses by density gradient centrifugation. Cells were either used directly after isolation for analysis of immune responses by ELIspot or frozen and stored in the gas phase of liquid nitrogen for later analyses.

In vitro stimulation ELIspot analysis. Freshly isolated PBMC were prestimulated with corresponding vaccine or control peptides $(5 \mu \mathrm{g} / \mathrm{ml})$ for 7 days. On day 7 , cells were counted and plated $\left(3 \times 10^{5} /\right.$ well) in anti-IFN $\gamma$ mAb precoated ELIspot-plates (MAHA S4510, 1-D1K, Mabtech, Stockholm). For the detection of antigen-specific $\mathrm{T}$ cells, $10 \mu \mathrm{g} / \mathrm{ml}$ of the respective vaccination peptides were added. As negative controls, cells were either left untreated or were stimulated with HIV-derived peptides (for HLA-A1: GSEELRSLY, and for HLA-A2: ILKEPVHGV). As positive control, cells were either stimulated with PHA $(5 \mu \mathrm{g} / \mathrm{ml}) / \mathrm{SEA}(20 \mathrm{ng} / \mathrm{ml})$ or with a mixture of Influenza, CMV, or EBV-derived peptides (for HLA-A1: VSDGGPNLY, CTELKLSDY, DSELEIKRY, LTEWGSGNRTY and for HLAA2: GILGFVFTL, CLGGLLTMV, LLDFVRFMGV, GLCTLVAML, NLVPMVATV), according to patient's HLA type. PHA (Phytohaemagglutinin) amd SEA (Staphylococcal Enterotoxin A) were both from Sigma, viral peptides were from Genscript. Following incubation at $37^{\circ} \mathrm{C}$ for 20 hours, wells were washed and stained with second $\mathrm{mAb}$ to IFN $\gamma$. ABC Vectastain Elite and 3-amino-9-ethylcarbazole were used to stain the membrane. Spots were counted using a computer-assisted video imaging analysis system (Carl Zeiss Vision). A response was considered positive if at least 2.5-fold above background staining and at least 10 spots were detectable.

Ex vivo class I peptide/HLA-tetramer staining. Thawed PBMC were sequentially stained with live/dead blue; 4 different peptide/HLA-tetramers labeled with PE, APC, BV421, or BV605; and surface-staining antibodies for CD3, CD4, CD8, CD45RA, and CD279/ PD1 (CD3 - SK7 - APC-H7, CD4 - SK3-BUV395, CD8-SK1 - PerCP, CD45RA- HI100- PE-Cy7, GranzymeB - AlexaFluor700, Perforin - $\delta$ G9- FITC and ki67 -B56- BV711 all from BD Bioscience). Then, cells were fixed, permeabilized, and intracellularly stained for GranzymeB, Perforin, and Ki67. Samples were analyzed on a BD FACS Fortessa. Examples of stainings and the gating strategy are shown in the supplementary information. The standard cut-off criteria for a positive response by pHLA-multimer staining were a minimum of 10 cells detected in the multimer-gate, a minimum percentage of $0.01 \%$ of the $\mathrm{CD}^{+} \mathrm{T}$ cells, and a population of the $\mathrm{pHLA}$-multimer positive cells that was clearly separated from the pHLA-multimer negative one.

Ex vivo class II peptide/HLA-tetramer staining. Thawed PBMC were sequentially stained with live/dead aqua, PE-labeled peptide/HLA-DR4 tetramer (SYLQDSVPDSFQD-HLA-DR4), and surface-staining antibodies to CD3, CD4, CD8, CD127, and CD14 (CD4-RPA-T4- V450, CD8 -SK1 PerCP, CD127-HIL7R-M21 - APC-Cy7 and CD14 -M5E2- PacificOrange, all from BD and FoxP3-PCH101- AlexaFluor700 from eBioscience). Then cells were fixed, permeabilized, and stained intracellularly with FoxP3-antibody. The analysis was performed on a BD FACS Canto. Examples of stainings are shown in Figure 3C.

Analysis of flow cytometry data. Data acquired with the FACS Calibur (MLPC limiting dilution tetramer readout) were analyzed with $\mathrm{BD}$ Cellquest software. FlowJo (9.1) was used to analyze data acquired from BD FACS Canto and Fortessa.

Quantification of intratumoral $T$ cells. To quantify $\mathrm{T}$ lymphocyte infiltrates in metastatic tumor tissues, 3- $\mu \mathrm{m}$ thick sections were cut from paraffin blocks and stained with H\&E. Immunohistochemical quantification of $\mathrm{T}$ lymphocytes was performed on anti-CD3-stained (Zytomed-Systems, clone SP7; dilution 1:150) sections according to a recently described method (The Cancer Genome Atlas Network 2015 , ref. 45). The resultant lymphocyte score (from 0-6) was generated as the sum of a lymphocyte distribution index and a lymphocyte density index. Each index ranged from $0-3$ points, adding up to a maximum lymphocyte score of 6 points. The lymphocyte distribution index differentiates between 0 , no lymphocytes within the tissue; 1 , lymphocytes present involving $<25 \%$ of the tissue cross-section area; 2 , lymphocytes present in $25 \%-50 \%$ of the tissue; 3 , lymphocytes present in $>50 \%$ of the tissue and the lymphocyte density index between 0 (absent), 1 (mild), 2 (moderate), and 3 (severe). Since the investigated tissue to a substantial portion represented lymph node metastases, care was taken to only count for tumor-infiltrating T lymphocytes and not for remainders of lymph node tissues.

Statistics. Statistical analysis was done using GraphPad Prism 6 software. If not stated otherwise, plots show the means with whiskers approximating the $95 \% \mathrm{CI}$ of the data. For statistical analysis, 2 -tailed $t$ tests with the assumption of inconsistent SD among samples and a false discovery rate approach of $1 \%$ were used. $P<0.05\left(^{*}\right), P<0.01\left({ }^{* *}\right)$, and $P<0.001\left({ }^{* *}\right)$ were considered statistically significant. 
Study approval. The trial was started following approval by the local IRB (Universitär Hospital Erlangen, Friedrich-Alexander-University Erlangen-Nuremberg (FAU), Erlangen, Germany) and the federal regulatory authorities (Paul Ehrlich Institute). All patients signed an informed consent.

\section{Author contributions}

GS and BST contributed equally to the trial design. BST was responsible for GMP manufacturing and clinical supervision of the trial. GS supervised the in vitro analyses. ME and IH were involved in patient care and performed the initial immunomonitoring analyses (ELIspot, MLPC). SV extended the MLPC assays to test the lytic capability and polyfunctionality of T cells with the help of PC, who was also an advisor for all other assays. SG further extended (tetramer stainings, MDSCs, Tregs, Luminex) the immunomonitoring and performed the final analyses. TB, E.Schultz, and LH were involved in patient care. LH and SS were involved in studying of the tumor microenvironment. KS independently evaluated the lymphocyte score. E. Strasser was responsible for the aphereses. PD and RJ evaluated the CT images. GS, BST, and SG wrote the manuscript, which was reviewed by all others.

\section{Acknowledgments}

We acknowledge the funding by the European Community, Sixth Framework Programme (Cancerimmunotherapy LSHC-CT-2006-518234; DC-THERA LSHB-CT-2004-512074), and the German Research Foundation (CRC 643, C1, Z2). We dedicate this paper to the loving memory of Ralph M. Steinman, who encouraged and helped us to perform this trial. We are grateful to all patients who participated in this trial. We thank (in alphabetical order) Ute Hirsch, Michaela May, Marita Rosenberg, Diane Stoica, and Manuel Wiesinger (GMP manufacturing); and Annett Haman, Waltraud Leisgang, Carmen Lorenz, and Manuel Wiesinger (immunomonitoring); the team of the cell sorting and immunomonitoring core unit Erlangen for excellent technical assistance; and the study nurses Doris Anzengruber and Stina Rosenheinrich.

Address correspondence to: Gerold Schuler, Department of Dermatology, University Hospital Erlangen, Internistisches Zentrum, Ulmenweg 18, D-91054 Erlangen, Germany. Phone: 49.9131.85.1010; E-mail: gerold.schuler@uk-erlangen.de.

IH's present address is: Fachbereich Dermatologie, Ambulantes Zentrum, Klinikum Bayreuth, Bayreuth, Germany.

SV's present address is: Advanced Medical Services GmbH, Mannheim, Germany.

TB's present address is: Health Bay Polyclinic, Dubai, United Arab Emirates.

ES's present address is: Department of Dermatology, General Hospital Nuremberg, Paracelsus Medical University, Nuremberg, Germany.

1. Romero P, et al. The Human Vaccines Project: A roadmap for cancer vaccine development. Sci Transl Med. 2016;8(334):334ps9.

2. van der Burg SH, Arens R, Ossendorp F, van Hall T, Melief CJ. Vaccines for established cancer: overcoming the challenges posed by immune evasion. Nat Rev Cancer. 2016;16(4):219-233.

3. van Elsas A, Hurwitz AA, Allison JP. Combination immunotherapy of B16 melanoma using anti-cytotoxic T lymphocyteassociated antigen 4 (CTLA-4) and granulocyte/macrophage colony-stimulating factor (GM-CSF)-producing vaccines induces rejection of subcutaneous and metastatic tumors accompanied by autoimmune depigmentation. J Exp Med. 1999;190(3):355-366.

4. Quezada SA, Peggs KS, Curran MA, Allison JP. CTLA4 blockade and GM-CSF combination immunotherapy alters the intratumor balance of effector and regulatory T cells. J Clin Invest. 2006;116(7):1935-1945.

5. Duraiswamy J, Kaluza KM, Freeman GJ, Coukos G. Dual blockade of PD-1 and CTLA-4 combined with tumor vaccine effectively restores T-cell rejection function in tumors. Cancer Res. 2013;73(12):3591-3603.

6. Soares KC, et al. PD-1/PD-L1 blockade together with vaccine therapy facilitates effector T-cell infiltration into pancreatic tumors. J Immunother. 2015;38(1):1-11.

7. Karyampudi L, et al. Accumulation of memory precursor CD8 T cells in regressing tumors following combination therapy with vaccine and anti-PD-1 antibody. Cancer Res. 2014;74(11):2974-2985.

8. Li B, VanRoey M, Wang C, Chen TH, Korman A, Jooss K. Anti-programmed death-1 synergizes with granulocyte macrophage colony-stimulating factor--secreting tumor cell immunotherapy providing therapeutic benefit to mice with established tumors. Clin Cancer Res. 2009;15(5):1623-1634.

9. Fu J, et al. STING agonist formulated cancer vaccines can cure established tumors resistant to PD-1 blockade. Sci Transl Med. 
2015;7(283):283ra52.

10. Le DT, et al. Evaluation of ipilimumab in combination with allogeneic pancreatic tumor cells transfected with a GM-CSF gene in previously treated pancreatic cancer. J Immunother. 2013;36(7):382-389.

11. Wilgenhof S, et al. Phase II Study of Autologous Monocyte-Derived mRNA Electroporated Dendritic Cells (TriMixDC-MEL) Plus Ipilimumab in Patients With Pretreated Advanced Melanoma. J Clin Oncol. 2016;34(12):1330-1338.

12. Hodi FS, et al. Immunologic and clinical effects of antibody blockade of cytotoxic T lymphocyte-associated antigen 4 in previously vaccinated cancer patients. Proc Natl Acad Sci USA. 2008;105(8):3005-3010.

13. Le DT, et al. PD-1 Blockade in Tumors with Mismatch-Repair Deficiency. N Engl J Med. 2015;372(26):2509-2520.

14. Van Allen EM, et al. Genomic correlates of response to CTLA-4 blockade in metastatic melanoma. Science. 2015;350(6257):207-211.

15. Johansson P, et al. Deep sequencing of uveal melanoma identifies a recurrent mutation in PLCB4. Oncotarget. 2016;7(4):4624-4631.

16. Steinman RM. Decisions about dendritic cells: past, present, and future. Annu Rev Immunol. 2012;30:1-22.

17. Palucka K, Banchereau J. Dendritic-cell-based therapeutic cancer vaccines. Immunity. 2013;39(1):38-48.

18. Small EJ, et al. Placebo-controlled phase III trial of immunologic therapy with sipuleucel-T (APC8015) in patients with metastatic, asymptomatic hormone refractory prostate cancer. J Clin Oncol. 2006;24(19):3089-3094.

19. Gulley JL, et al. Perspectives on sipuleucel-T: Its role in the prostate cancer treatment paradigm. Oncoimmunology. 2016;5(4):e1107698.

20. Kantoff PW, et al. Sipuleucel-T immunotherapy for castration-resistant prostate cancer. $N$ Engl J Med. 2010;363(5):411-422.

21. Thurner B, et al. Generation of large numbers of fully mature and stable dendritic cells from leukapheresis products for clinical application. J Immunol Methods. 1999;223(1):1-15.

22. Erdmann M, et al. Effective clinical-scale production of dendritic cell vaccines by monocyte elutriation directly in medium, subsequent culture in bags and final antigen loading using peptides or RNA transfection. J Immunother. 2007;30(6):663-674.

23. Dhodapkar MV, et al. Rapid generation of broad T-cell immunity in humans after a single injection of mature dendritic cells. $J$ Clin Invest. 1999;104(2):173-180.

24. Thurner B, et al. Vaccination with mage-3A1 peptide-pulsed mature, monocyte-derived dendritic cells expands specific cytotoxic T cells and induces regression of some metastases in advanced stage IV melanoma. $J$ Exp Med. 1999;190(11):1669-1678.

25. Dhodapkar MV, Krasovsky J, Steinman RM, Bhardwaj N. Mature dendritic cells boost functionally superior CD8(+) T-cell in humans without foreign helper epitopes. J Clin Invest. 2000;105(6):R9-R14.

26. Schuler-Thurner B, et al. Rapid induction of tumor-specific type $1 \mathrm{~T}$ helper cells in metastatic melanoma patients by vaccination with mature, cryopreserved, peptide-loaded monocyte-derived dendritic cells. J Exp Med. 2002;195(10):1279-1288.

27. Sallusto F, Lanzavecchia A. Efficient presentation of soluble antigen by cultured human dendritic cells is maintained by granulocyte/macrophage colony-stimulating factor plus interleukin 4 and downregulated by tumor necrosis factor alpha. J Exp Med. 1994;179(4):1109-1118.

28. Cella M, Scheidegger D, Palmer-Lehmann K, Lane P, Lanzavecchia A, Alber G. Ligation of CD40 on dendritic cells triggers production of high levels of interleukin-12 and enhances T cell stimulatory capacity: T-T help via APC activation. J Exp Med. 1996;184(2):747-752.

29. Lanzavecchia A. Immunology. Licence to kill. Nature. 1998;393(6684):413-414.

30. Ma DY, Clark EA. The role of CD40 and CD154/CD40L in dendritic cells. Semin Immunol. 2009;21(5):265-272.

31. Pinto S, et al. Misinitiation of intrathymic MART-1 transcription and biased TCR usage explain the high frequency of MART1-specific T cells. Eur J Immunol. 2014;44(9):2811-2821.

32. Romero P, Speiser DE, Rufer N. Deciphering the unusual HLA-A2/Melan-A/MART-1-specific TCR repertoire in humans. Eur J Immunol. 2014;44(9):2567-2570.

33. Schuler-Thurner B, et al. Mage-3 and influenza-matrix peptide-specific cytotoxic T cells are inducible in terminal stage HLAA2.1+ melanoma patients by mature monocyte-derived dendritic cells. J Immunol. 2000;165(6):3492-3496.

34. Feuerstein B, et al. A method for the production of cryopreserved aliquots of antigen-preloaded, mature dendritic cells ready for clinical use. J Immunol Methods. 2000;245(1-2):15-29.

35. Kuniyoshi JS, et al. Dendritic cell secretion of IL-15 is induced by recombinant huCD40LT and augments the stimulation of antigen-specific cytolytic T cells. Cell Immunol. 1999;193(1):48-58

36. Kalady MF, Onaitis MW, Emani S, Abdul-Wahab Z, Pruitt SK, Tyler DS. Dendritic cells pulsed with pancreatic cancer total tumor RNA generate specific antipancreatic cancer T cells. J Gastrointest Surg. 2004;8(2):175-81; discussion 181.

37. Terheyden P, Straten P, Bröcker EB, Kämpgen E, Becker JC. CD40-ligated dendritic cells effectively expand melanoma-specific CD8+ CTLs and CD4+ IFN-gamma-producing T cells from tumor-infiltrating lymphocytes. J Immunol. 2000;164(12):6633-6639.

38. Millard AL, Ittelet D, Schooneman F, Bernard J. Dendritic cell KLH loading requirements for efficient CD4+ T-cell priming and help to peptide-specific cytotoxic T-cell response, in view of potential use in cancer vaccines. Vaccine. 2003;21(9-10):869-876.

39. Becht E, et al. Immune Contexture, Immunoscore, and Malignant Cell Molecular Subgroups for Prognostic and Theranostic Classifications of Cancers. Adv Immunol. 2016;130:95-190.

40. Angell H, Galon J. From the immune contexture to the Immunoscore: the role of prognostic and predictive immune markers in cancer. Curr Opin Immunol. 2013;25(2):261-267.

41. Clemente CG, Mihm MC, Bufalino R, Zurrida S, Collini P, Cascinelli N. Prognostic value of tumor infiltrating lymphocytes in the vertical growth phase of primary cutaneous melanoma. Cancer. 1996;77(7):1303-1310.

42. Mihm MC, Clemente CG, Cascinelli N. Tumor infiltrating lymphocytes in lymph node melanoma metastases: a histopathologic prognostic indicator and an expression of local immune response. Lab Invest. 1996;74(1):43-47.

43. Azimi F, et al. Tumor-infiltrating lymphocyte grade is an independent predictor of sentinel lymph node status and survival in patients with cutaneous melanoma. J Clin Oncol. 2012;30(21):2678-2683.

44. Vasaturo A, et al. T-cell Landscape in a Primary Melanoma Predicts the Survival of Patients with Metastatic Disease after Their Treatment with Dendritic Cell Vaccines. Cancer Res. 2016;76(12):3496-3506.

45. Cancer Genome Atlas Network. Genomic Classification of Cutaneous Melanoma. Cell. 2015;161(7):1681-1696.

46. Gajewski TF, Fuertes M, Spaapen R, Zheng Y, Kline J. Molecular profiling to identify relevant immune resistance mechanisms 
in the tumor microenvironment. Curr Opin Immunol. 2011;23(2):286-292.

47. Hamid O, et al. A prospective phase II trial exploring the association between tumor microenvironment biomarkers and clinical activity of ipilimumab in advanced melanoma. J Transl Med. 2011;9:204.

48. Ji RR, et al. An immune-active tumor microenvironment favors clinical response to ipilimumab. Cancer Immunol Immunother. 2012;61(7):1019-1031

49. Spranger S, Bao R, Gajewski TF. Melanoma-intrinsic $\beta$-catenin signalling prevents anti-tumour immunity. Nature. 2015;523(7559):231-235.

50. Delyon J, Maio M, Lebbé C. The ipilimumab lesson in melanoma: achieving long-term survival. Semin Oncol. 2015;42(3):387-401.

51. Schadendorf D, et al. Pooled Analysis of Long-Term Survival Data From Phase II and Phase III Trials of Ipilimumab in Unresectable or Metastatic Melanoma. J Clin Oncol. 2015;33(17):1889-1894.

52. Wilgenhof S, et al. Long-term clinical outcome of melanoma patients treated with messenger RNA-electroporated dendritic cell therapy following complete resection of metastases. Cancer Immunol Immunother. 2015;64(3):381-388.

53. Bol KF, et al. Favorable overall survival in stage III melanoma patients after adjuvant dendritic cell vaccination. Oncoimmunology. 2016;5(1):e1057673.

54. Gibney GT, et al. Safety, correlative markers, and clinical results of adjuvant nivolumab in combination with vaccine in resected high-risk metastatic melanoma. Clin Cancer Res. 2015;21(4):712-720.

55. Eggermont AM, et al. Prolonged Survival in Stage III Melanoma with Ipilimumab Adjuvant Therapy. $N$ Engl J Med. 2016;375(19):1845-1855.

56. Connerotte T, et al. Functions of Anti-MAGE T-cells induced in melanoma patients under different vaccination modalities. Cancer Res. 2008;68(10):3931-3940.

57. Godelaine D, et al. Polyclonal CTL responses observed in melanoma patients vaccinated with dendritic cells pulsed with a MAGE-3.A1 peptide. J Immunol. 2003;171(9):4893-4897.

58. Wimmers F, et al. Long-lasting multifunctional CD8(+) T cell responses in end-stage melanoma patients can be induced by dendritic cell vaccination. Oncoimmunology. 2016;5(1):e1067745.

59. Gaucher D, et al. Yellow fever vaccine induces integrated multilineage and polyfunctional immune responses. J Exp Med. 2008;205(13):3119-3131.

60. Betts MR, et al. HIV nonprogressors preferentially maintain highly functional HIV-specific CD8+ T cells. Blood. 2006;107(12):4781-4789.

61. Lévy Y, et al. Dendritic cell-based therapeutic vaccine elicits polyfunctional HIV-specific T-cell immunity associated with control of viral load. Eur J Immunol. 2014;44(9):2802-2810.

62. Marshall NA, Galvin KC, Corcoran AM, Boon L, Higgs R, Mills KH. Immunotherapy with PI3K inhibitor and Toll-like receptor agonist induces IFN- $\gamma+\mathrm{IL}-17+$ polyfunctional T cells that mediate rejection of murine tumors. Cancer Res. 2012;72(3):581-591.

63. Ma C, et al. Multifunctional T-cell analyses to study response and progression in adoptive cell transfer immunotherapy. Cancer Discov. 2013;3(4):418-429.

64. Yuan J, et al. CTLA-4 blockade enhances polyfunctional NY-ESO-1 specific T cell responses in metastatic melanoma patients with clinical benefit. Proc Natl Acad Sci USA. 2008;105(51):20410-20415.

65. Kalady MF, Onaitis MW, Emani S, Abdel-Wahab Z, Tyler DS, Pruitt SK. Sequential delivery of maturation stimuli increases human dendritic cell IL-12 production and enhances tumor antigen-specific immunogenicity. J Surg Res. 2004;116(1):24-31.

66. Hoyer S, et al. Concurrent interaction of DCs with CD4(+) and CD8(+) T cells improves secondary CTL expansion: It takes three to tango. Eur J Immunol. 2014;44(12):3543-3559.

67. Pfeiffer IA, et al. Triggering of NF-אB in cytokine-matured human DCs generates superior DCs for T-cell priming in cancer immunotherapy. Eur J Immunol. 2014;44(11):3413-3428.

68. Fay JW, et al. Long-term outcomes in patients with metastatic melanoma vaccinated with melanoma peptide-pulsed CD34(+) progenitor-derived dendritic cells. Cancer Immunol Immunother. 2006;55(10):1209-1218

69. Dillman RO, et al. Dendritic Versus Tumor Cell Presentation of Autologous Tumor Antigens for Active Specific Immunotherapy in Metastatic Melanoma: Impact on Long-Term Survival by Extent of Disease at the Time of Treatment. Cancer Biother Radiopharm. 2015;30(5):187-194.

70. O'Rourke MG, et al. Durable complete clinical responses in a phase I/II trial using an autologous melanoma cell/dendritic cell vaccine. Cancer Immunol Immunother. 2003;52(6):387-395

71. O'Rourke MG, et al. Dendritic cell immunotherapy for stage IV melanoma. Melanoma Res. 2007;17(5):316-322.

72. Ridolfi L, et al. Dendritic cell-based vaccine in advanced melanoma: update of clinical outcome. Melanoma Res. 2011;21(6):524-529.

73. Ellebaek E, et al. Metastatic melanoma patients treated with dendritic cell vaccination, Interleukin-2 and metronomic cyclophosphamide: results from a phase II trial. Cancer Immunol Immunother. 2012;61(10):1791-1804.

74. Trepiakas R, et al. Vaccination with autologous dendritic cells pulsed with multiple tumor antigens for treatment of patients with malignant melanoma: results from a phase I/II trial. Cytotherapy. 2010;12(6):721-734

75. Aarntzen EH, et al. Targeting CD4(+) T-helper cells improves the induction of antitumor responses in dendritic cell-based vaccination. Cancer Res. 2013;73(1):19-29.

76. Aarntzen EH, et al. Vaccination with mRNA-electroporated dendritic cells induces robust tumor antigen-specific CD4+ and CD8+ T cells responses in stage III and IV melanoma patients. Clin Cancer Res. 2012;18(19):5460-5470.

77. Wilgenhof S, et al. Therapeutic vaccination with an autologous mRNA electroporated dendritic cell vaccine in patients with advanced melanoma. J Immunother. 2011;34(5):448-456.

78. Oshita C, et al. Dendritic cell-based vaccination in metastatic melanoma patients: phase II clinical trial. Oncol Rep. 2012;28(4):1131-1138.

79. Palucka K, Ueno H, Zurawski G, Fay J, Banchereau J. Building on dendritic cell subsets to improve cancer vaccines. Curr Opin Immunol. 2010;22(2):258-263.

80. Dillman RO, et al. Phase II trial of dendritic cells loaded with antigens from self-renewing, proliferating autologous tumor cells as patient-specific antitumor vaccines in patients with metastatic melanoma: final report. Cancer Biother Radiopharm. 
2009;24(3):311-319

81. Pritchard AL, et al. Exome Sequencing to Predict Neoantigens in Melanoma. Cancer Immunol Res. 2015;3(9):992-998.

82. McNeel DG, et al. A transient increase in eosinophils is associated with prolonged survival in men with metastatic castrationresistant prostate cancer who receive sipuleucel-T. Cancer Immunol Res. 2014;2(10):988-999.

83. Gebhardt C, et al. Myeloid Cells and Related Chronic Inflammatory Factors as Novel Predictive Markers in Melanoma Treatment with Ipilimumab. Clin Cancer Res. 2015;21(24):5453-5459.

84. Martens A, et al. Baseline Peripheral Blood Biomarkers Associated with Clinical Outcome of Advanced Melanoma Patients Treated with Ipilimumab. Clin Cancer Res. 2016;22(12):2908-2918.

85. Delyon J, et al. Experience in daily practice with ipilimumab for the treatment of patients with metastatic melanoma: an early increase in lymphocyte and eosinophil counts is associated with improved survival. Ann Oncol. 2013;24(6):1697-1703.

86. Simson L, et al. Regulation of carcinogenesis by IL-5 and CCL11: a potential role for eosinophils in tumor immune surveillance. J Immunol. 2007;178(7):4222-4229.

87. Costain DJ, Guha AK, Liwski RS, Lee TD. Murine hypodense eosinophils induce tumour cell apoptosis by a granzyme B-dependent mechanism. Cancer Immunol Immunother. 2001;50(6):293-299.

88. Ikutani M, et al. Identification of innate IL-5-producing cells and their role in lung eosinophil regulation and antitumor immunity. J Immunol. 2012;188(2):703-713.

89. Legrand $\mathrm{F}$, et al. Human eosinophils exert TNF- $\alpha$ and granzyme A-mediated tumoricidal activity toward colon carcinoma cells. J Immunol. 2010;185(12):7443-7451.

90. Rivoltini L, et al. In vitro anti-tumor activity of eosinophils from cancer patients treated with subcutaneous administration of interleukin 2. Role of interleukin 5. Int J Cancer. 1993;54(1):8-15.

91. Gatault S, et al. IL-18 Is Involved in Eosinophil-Mediated Tumoricidal Activity against a Colon Carcinoma Cell Line by Upregulating LFA-1 and ICAM-1. J Immunol. 2015;195(5):2483-2492.

92. Carretero R, Sektioglu IM, Garbi N, Salgado OC, Beckhove P, Hämmerling GJ. Eosinophils orchestrate cancer rejection by normalizing tumor vessels and enhancing infiltration of CD8(+) T cells. Nat Immunol. 2015;16(6):609-617.

93. Prizment AE, et al. Tumor eosinophil infiltration and improved survival of colorectal cancer patients: Iowa Women's Health Study. Mod Pathol. 2016;29(5):516-527.

94. Sakkal S, Miller S, Apostolopoulos V, Nurgali K. Eosinophils in Cancer: Favourable or Unfavourable? Curr Med Chem. 2016;23(7):650-666.

95. Soiffer R, et al. Vaccination with irradiated autologous melanoma cells engineered to secrete human granulocyte-macrophage colony-stimulating factor generates potent antitumor immunity in patients with metastatic melanoma. Proc Natl Acad Sci USA. 1998;95(22):13141-13146.

96. Schrama D, et al. Aggregation of antigen-specific T cells at the inoculation site of mature dendritic cells. J Invest Dermatol. 2002;119(6):1443-1448.

97. de Vries IJ, et al. Immunomonitoring tumor-specific T cells in delayed-type hypersensitivity skin biopsies after dendritic cell vaccination correlates with clinical outcome. J Clin Oncol. 2005;23(24):5779-5787.

98. Nakai N, et al. Immunohistological analysis of peptide-induced delayed-type hypersensitivity in advanced melanoma patients treated with melanoma antigen-pulsed mature monocyte-derived dendritic cell vaccination. J Dermatol Sci. 2009;53(1):40-47.

99. Engell-Noerregaard L, Hansen TH, Andersen MH, Thor Straten P, Svane IM. Review of clinical studies on dendritic cell-based vaccination of patients with malignant melanoma: assessment of correlation between clinical response and vaccine parameters. Cancer Immunol Immunother. 2009;58(1):1-14.

100. Nakai N, Hartmann G, Kishimoto S, Katoh N. Dendritic cell vaccination in human melanoma: relationships between clinical effects and vaccine parameters. Pigment Cell Melanoma Res. 2010;23(5):607-619.

101. Boudewijns S, et al. Immune-related Adverse Events of Dendritic Cell Vaccination Correlate With Immunologic and Clinical Outcome in Stage III and IV Melanoma Patients. J Immunother. 2016;39(6):241-248.

102. Aarntzen EH, et al. Skin-test infiltrating lymphocytes early predict clinical outcome of dendritic cell-based vaccination in metastatic melanoma. Cancer Res. 2012;72(23):6102-6110.

103. Kvistborg P, et al. Anti-CTLA-4 therapy broadens the melanoma-reactive CD8+ T cell response. Sci Transl Med. 2014;6(254):254ra128.

104. Boddupalli CS, et al. Interlesional diversity of T cell receptors in melanoma with immune checkpoints enriched in tissue-resident memory T cells. JCI Insight. 2016;1(21):e88955.

105. Lurquin C, et al. Contrasting frequencies of antitumor and anti-vaccine $\mathrm{T}$ cells in metastases of a melanoma patient vaccinated with a MAGE tumor antigen. $J$ Exp Med. 2005;201(2):249-257.

106. Carrasco J, et al. Vaccination of a melanoma patient with mature dendritic cells pulsed with MAGE-3 peptides triggers the activity of nonvaccine anti-tumor cells. J Immunol. 2008;180(5):3585-3593.

107. Corbière V, et al. Antigen spreading contributes to MAGE vaccination-induced regression of melanoma metastases. Cancer Res. 2011;71(4):1253-1262.

108. Schadendorf D, et al. Dacarbazine (DTIC) versus vaccination with autologous peptide-pulsed dendritic cells (DC) in first-line treatment of patients with metastatic melanoma: a randomized phase III trial of the DC study group of the DeCOG. Ann Oncol. 2006;17(4):563-570.

109. Kennedy R, Celis E. Multiple roles for CD4+ T cells in anti-tumor immune responses. Immunol Rev. 2008;222:129-144.

110. Wong SB, Bos R, Sherman LA. Tumor-specific CD4+ T cells render the tumor environment permissive for infiltration by lowavidity CD8+ T cells. J Immunol. 2008;180(5):3122-3131.

111. Braumüller H, et al. T-helper-1-cell cytokines drive cancer into senescence. Nature. 2013;494(7437):361-365.

112. Schultz ES, et al. Functional analysis of tumor-specific Th cell responses detected in melanoma patients after dendritic cellbased immunotherapy. J Immunol. 2004;172(2):1304-1310.

113. Hunder NN, et al. Treatment of metastatic melanoma with autologous CD4+ T cells against NY-ESO-1. NEngl J Med. 2008;358(25):2698-2703.

114. Tran E, et al. Cancer immunotherapy based on mutation-specific CD4+ T cells in a patient with epithelial cancer. Science. 
2014;344(6184):641-645.

115. Shahabi V, Postow MA, Tuck D, Wolchok JD. Immune-priming of the tumor microenvironment by radiotherapy: rationale for combination with immunotherapy to improve anticancer efficacy. Am J Clin Oncol. 2015;38(1):90-97.

116. Gajewski T, Zha Y, Thurner B, Schuler G. Association of gene expression profile in melanoma and survival to a dendritic cellbased vaccine. J Clin Oncol. 2009;27(Suppl 15):9002.

117. Gilboa E. DC-based cancer vaccines. J Clin Invest. 2007;117(5):1195-1203.

118. Osada T, et al. Precision cancer immunotherapy: optimizing dendritic cell-based strategies to induce tumor antigen-specific T-cell responses against individual patient tumors. J Immunother. 2015;38(4):155-164.

119. Janetzki S, et al. "MIATA"-minimal information about T cell assays. Immunity. 2009;31(4):527-528.

120. Romani N, et al. Generation of mature dendritic cells from human blood. An improved method with special regard to clinical applicability. J Immunol Methods. 1996;196(2):137-151.

121. Jonuleit $\mathrm{H}$, et al. Pro-inflammatory cytokines and prostaglandins induce maturation of potent immunostimulatory dendritic cells under fetal calf serum-free conditions. Eur J Immunol. 1997;27(12):3135-3142. 